\title{
Low-Complexity Iterative Detection for Dual-Mode Index Modulation in Dispersive Nonlinear Satellite Channels
}

\author{
Qiaolin Shi, Student Member, IEEE, Nan Wu, Member, IEEE, Diep N. Nguyen, Senior Member, IEEE, Xiaojing \\ Huang, Senior Member, IEEE, Hua Wang, Member, IEEE, Lajos Hanzo, Fellow, IEEE
}

\begin{abstract}
The integration of terrestrial and satellite communications (Satcom) is advocated for satisfying the challenging requirements of seamless, high-performance services. However, both the bandwidth and the power available are limited over satellite channels. In this paper, we propose index modulation (IM) and code-aided Satcom by conveying information by a pair of distinguishable constellation modes and their permutations. In order to combat both the linear and nonlinear distortion imposed by satellite channels, we conceive a factor graph (FG)based iterative detection algorithm for Satcom relying on dualmode (DM) IM (Sat-DMIM). The correlation amongst Sat-DMIM symbols imposed by both the channel-induced dispersion and the mode-selection mapping is explicitly represented by the FG constructed. Then the amalgamated belief propagation (BP) and mean field (MF) message passing algorithm is derived over this FG for detecting both the IM bits and the classic constellation mapping bits, while eliminating both the linear and nonlinear distortions. The complexity of the iterative detection algorithm is reduced by linearizing some high-order terms appearing in nonlinear distortion components using the a posteriori estimates of the Sat-DMIM symbols obtained from the previous iteration. Our simulation results demonstrate the power of the proposed amalgamated BP-MF-based and partial linearization approximation-based iterative detection algorithms.
\end{abstract}

Index Terms-Nonlinear dispersive satellite channels, dual mode, index modulation (IM), satellite communications, factor graph, iterative/turbo detection.

\section{INTRODUCTION}

Satellite communications (Satcom) can provide economical network access in remote areas to reliable services, including emergency communications. Hence next-generation terrestrial wireless systems are expected to cooperate with Satcom networks for providing seamless global coverage [1]-[5]. In order to keep up with the rapid growth of terrestrial communications, Satcom should exploit innovative techniques to improve its spectral- vs. energy-efficiency trade-off.

Recently, index modulation (IM) has been widely investigated as a strong candidate for future terrestrial cellular systems, due to its unique advantages in terms of bandwidth-

This work was supported by the National Science Foundation of China under Grant 61971041 and Grant 62001027. L. Hanzo would like to acknowledge the financial support of the Engineering and Physical Sciences Research Council projects EP/P034284/1 and EP/P003990/1 (COALESCE) as well as of the European Research Council's Advanced Fellow Grant QuantCom (Grant No. 789028). (Corresponding author: Nan Wu.)

Q. Shi was with the School of Information and Electronics, Beijing Institute of Technology, 100081, China, and with Faculty of Engineering and IT, University of Technology Sydney, Ultimo, NSW 2007, Australia (e-mail: qiaolin.shi@alumni.uts.edu.au).

$\mathrm{N}$. Wu and $\mathrm{H}$. Wang are with the School of Information and Electronics, Beijing Institute of Technology, 100081, China (e-mail: \{wunan, wanghua $@ @$ bit.edu.cn).

D. Nguyen and X. Huang are with Faculty of Engineering and IT, University of Technology Sydney, Ultimo, NSW 2007, Australia (e-mail: \{diep.nguyen, xiaojing.huang\}@uts.edu.au).

L. Hanzo is with the School of Electronics and Computer Science, University of Southampton, Southampton, SO17 1BJ, U.K. (e-mail: lh@ecs.soton.ac.uk). and power-efficiency [6]-[10]. In contrast to other popular spectrum-efficient techniques, such as the non-orthogonal transmission [4], [5], [11], IM introduces a new dimension for transmitting information. By exploiting the indices of the building blocks, additional IM information bits are embedded into the transmitted signals without consuming additional power [6]. The power conserved may then be reassigned to the classic constellation symbols, which leads to an improved bit error ratio (BER) performance compared to the traditional schemes under the same total transmission energy [12].

The IM concept applied in the spatial domain is termed as spatial modulation (SM), where information is conveyed not only by the classic amplitude phase shift keying (APSK)/quadrature amplitude modulation (QAM) constellation symbols but also by the indices of active transmit antennas (TA) [7]. Inspired by SM, orthogonal frequency division multiplexing (OFDM) with IM (OFDM-IM) has been developed and attracted wide spread attention due to its appealing advantages (e.g., higher energy efficiency and improved BER performance) over classical OFDM [8], [13], [14]. However, OFDM-IM also inherits the drawbacks of OFDM, including its high peak-to-average power ratio (PAPR) and the vulnerability to time-varying multipath propagation. For circumventing these problems of multi-carrier systems while enjoying the advantages of IM, the broadband single-carrier (SC)-based IM (SC-IM) concept was proposed in [9] and further enhanced in [15] by exploiting faster-than-Nyquist signaling. In [10], the SC-IM scheme was shown to be particularly beneficial for Internet of Things (IoT) devices, which have stringent constraints and limitations, since the complexity of the SCIM transmitter of IoT devices is expected to be lower than that of OFDM.

However, the spectral efficiency of the aforementioned IM schemes remains limited due to the existence of idle antennas/subcarriers/time slots. In order to overcome this limitation of IM, dual-mode (DM) IM (DMIM) was introduced in [16][18] and its improved version - multiple-mode (MM) IM (MMIM) - was introduced in [19], [20]. The DMIM scheme, unlike its single-mode counterpart, conveys symbols drawn from a secondary constellation represented by the inactive transmit entities of the original IM. By designing the primary and secondary constellations to ensure that they can be distinguished from each other, the DMIM scheme can achieve a higher minimum Euclidean distance (MED) over its singlemode counterpart [17], [21].

Although various IM techniques have attracted considerable research attention in terrestrial networks, their application in dispersive, nonlinear Satcom channels is a challenging new 
topic. Indeed, at the time of writing no journal contributions have been published on this subject. We note that SM [22], [23] is not suitable for Satcom, where the transmitter-toreceiver satellite links are not sufficiently unique for them to be distinguishable amongst users. This means that data communication might be impossible since the signals emitted by the TA at the altitude of satellites will look approximately the same at the extremely distant receiver [24]. On the other hand, although both OFDM and SC modulations have been widely employed in Satcom, the receiver design of IMaided OFDM and SC becomes more challenging in Satcom. In contrast to existing IM-based terrestrial communication systems, which typically operate in linear frequency-flat [7] or linear frequency-selective Rayleigh fading channels [8], [9], [15], Satcom generally suffers both from linear dispersion and nonlinear distortions [25]. Consequently, the receiver has to combat both the dispersion-induced linear distortions inflicted by the inter-symbol interference (ISI) and nonlinear distortions, as well as to jointly detect the IM bits and the classic APSK/QAM bits. To the best of our knowledge, iterative detection of IM in nonlinear dispersive satellite channels has not been studied.

In Satcom without using IM, many algorithms have been developed for SC signal detection [26]-[30]. SC modulation typically has low PAPR and hence it is less vulnerable to nonlinear distortions than OFDM [31]. In [26], a lowcomplexity detector based on Gaussian approximation of the nonlinear terms was developed for SC in Satcom. However, it exhibits a gravely degraded performance compared to the optimal receiver. In [27], the sum-product algorithm (SPA) was employed for detecting PSK symbols relying on factor graphs (FG) [32]. The complexity of this detector increases only linearly with the nonlinear channel's memory, albeit at the cost of significant performance loss. By using the Markov chain Monte Carlo (MCMC) technique, a nonlinear equalizer was studied in [33], which offers an attractive tradeoff between the BER performance and the complexity.

Unfortunately, many of the existing detectors can not be directly applied in Satcom relying on IM. One of the reasons is that not all permutations of the two distinguishable constellation modes ${ }^{1}$ are utilized by the transmitter, and this has to be carefully taken into account at the receiver. Moreover, the discrete constellation symbols are not independent due to the use of IM, which is not the case in conventional Satcom. In this context, the widely-used independent Gaussian approximation of the discrete symbol variables of conventional Satcom will lead to significant performance loss when directly used in IMaided Satcom. On the other hand, the impairments imposed by satellite channels destroy the orthogonality of the indexmodulated symbol vector (IM-symbol). If we want to restore their orthogonality, the complexity of the optimal detector will increase exponentially with the number of bits per frame [8].

Against this background, we conceive the DMIM technique for improving the spectral efficiency of single-carrier Satcom. Then we develop a pair of low-complexity iterative detection

\footnotetext{
${ }^{1}$ Single-mode-aided IM can be regarded as a special case of DMIM by setting the secondary constellation to be null.
}

algorithms for Satcom relying on DMIM (Sat-DMIM) for dispersive nonlinear satellite channels. The contributions of this paper are summarized as follows:

- Instead of directly treating the correlated Sat-DMIM symbols as independent Gaussian random variables, we take full account of the dual-mode selection pattern constraint by explicitly calculating the a priori probability density function (pdf) of the Sat-DMIM signal. Given this $a$ priori information, the joint a posteriori pdf of discrete Sat-DMIM symbols is derived based on a Volterra-series expansion model [34] for dispersive nonlinear satellite channels.

- In order to eliminate the effects of both the linear and nonlinear distortion of satellite channels, we construct a suitably-designed factor graph by factorizing the joint a posteriori probability distribution, based on which an amalgamated belief propagation (BP) and mean field (MF) message passing algorithm is derived. Specifically, the MF method is applied at the observation nodes for decoupling the Sat-DMIM symbols, while the BP technique is invoked at both the mode-selection pattern constraint nodes and at the PSK/QAM modulation constraint nodes for updating the estimates of the IM bits and APSK/QAM bits.

- In contrast to directly approximating the nonlinear system model [27], [35], [36], we propose a partial linearization approximation-aided BP-MF algorithm for further reducing the complexity. Specifically, by ignoring some highorder ISI terms, the message parameters to be updated throughout the iterations are simplified. The extent of sufficient statistics to be tracked for reliable detection is further reduced by using the first-order Taylor series expansion technique.

The rest of this paper is outlined as follows. Section II introduces the system model. Low-complexity BP-MF-based and partial linearization approximation-based iterative detection algorithms are derived over a suitably-designed factor graph in Section III and Section IV, respectively. Section V provides our computer simulation results and complexity analysis. Finally, Section VI concludes this paper.

Notation: $X_{m, n}$ and $x_{n}$ denote the $(m, n)$ th and the $n$th entries of matrix $\mathbf{X}$ and vector $\mathbf{x}$, respectively; $\mathbb{R}$ and $\mathbb{C}$ are the sets of real and complex numbers, respectively; Superscript ${ }^{T}$ stands for the transpose operation; $\left(\begin{array}{l}n \\ k\end{array}\right)$ represents the binomial coefficient; $\lfloor\cdot\rfloor$ indicates the floor operation; $\mathcal{N}_{c}\left(x ; \hat{m}_{x}, \hat{v}_{x}\right)$ denotes a Gaussian distribution of random variable $x$ with mean $\hat{m}_{x}$ and variance $\hat{v}_{x}$, the notation $\propto$ represents equality of functions up to a positive constant, $E(\cdot)_{b(x)}$ is the expectation operation with respect to $x$ given the condition function $b(x), \Re\{x\}$ and $\Im\{x\}$ are the real and imaginary parts of $x$, respectively. The notation $\triangleq$ represents the perfect equivalence of functions.

\section{SySTEM MODEL}

We consider a burst-mode based transmission, which is a typical scenario in Satcom. The transceiver architecture of lowdensity parity-check (LDPC)-coded [37] Satcom relying on 

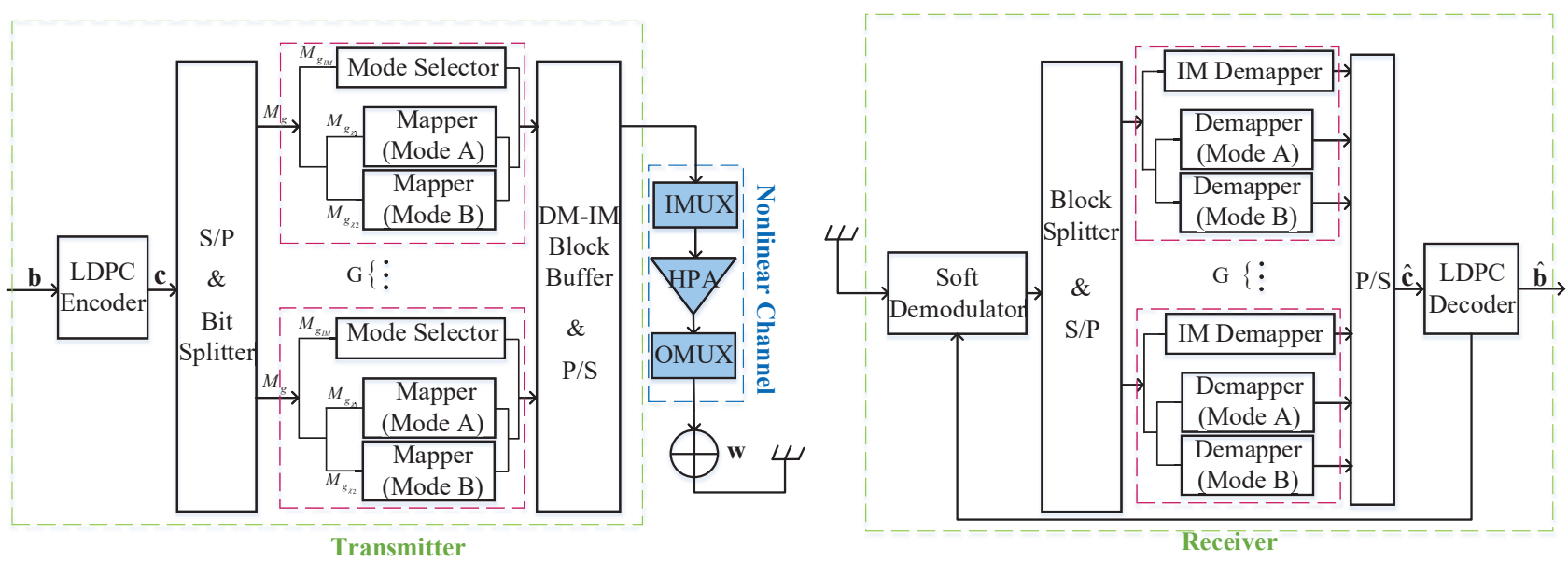

Fig. 1. The transceiver of the LDPC-coded Satcom relying on DMIM.

TABLE I

THE LOOK-UP TABLE OF MODE-SELECTION MAPPING BETWEEN THE IM BITS AND THE LEGITIMATE SET FOR $N_{g}=4$ AND $K_{g}=2$.

\begin{tabular}{|c|c|c|}
\hline$M_{g_{\mathrm{I}}}$ & $\mathcal{I}_{A}$ & $\mathbf{x}_{g}$ \\
\hline$[00]$ & $\{1,3\}$ & {$\left[s_{q}^{A}(1), s_{q}^{B}(1), s_{q}^{A}(2), s_{q}^{B}(2)\right]^{T}$} \\
\hline$\left[\begin{array}{ll}0 & 1\end{array}\right]$ & $\{1,4\}$ & {$\left[s_{q}^{A}(1), s_{q}^{B}(1), s_{q}^{B}(2), s_{q}^{A}(2)\right]^{T}$} \\
\hline$[10]$ & $\{2,3\}$ & {$\left[s_{g}^{B}(1), s_{g}^{A}(1), s_{g}^{A}(2), s_{g}^{B}(2)\right]^{T}$} \\
\hline$[11]$ & $\{2,4\}$ & {$\left[s_{q}^{B}(1), s_{q}^{A}(1), s_{q}^{B}(2), s_{q}^{A}(2)\right]^{T}$} \\
\hline
\end{tabular}

DMIM is depicted in Fig. 1. For each Sat-DMIM frame, the $N$-length symbols vectors $\mathbf{x}=\left[x_{1}, x_{2}, \ldots, x_{N}\right]^{T}$ are equally partitioned into $G$ subframes, i.e., $\mathbf{x} \triangleq\left[\mathbf{x}_{1}^{T}, \ldots, \mathbf{x}_{G}^{T}\right]^{T}$ with each subframe containing $N_{g} \triangleq N / G$ constellation symbols. In contrast to conventional single-carrier Satcom, where only a single constellation mode is employed for all time slots, the $N_{g}$ symbols in each Sat-DMIM subframe are drawn from a pair of distinguishable constellation modes. Therefore, in Sat-DMIM, the information is conveyed both by conventional PSK/QAM symbols and by the permutation of the two modes predesigned for each subframe. The details of the transmitter and the receiver structures, as well as the characteristics of nonlinear dispersive satellite channels are provided in the following subsections.

\section{A. Transmitter}

As shown in Fig. 1, a length- $M_{i}$ sequence of information bits $\mathbf{b} \triangleq\left\{b_{i}\right\}_{i=1}^{M_{i}}$ is encoded by a rate- $R_{\mathrm{c}}$ LDPC encoder, and then the resultant $M_{c} \triangleq M_{i} / R_{\mathrm{c}}$ coded bits $\mathbf{c}=\left\{c_{i}\right\}_{i=1}^{M_{c}}$ are equally partitioned into $G$ number of length- $M_{g}\left(M_{g} \triangleq M_{c} / G\right)$ subgroups, i.e., we have $\mathbf{c} \triangleq\left[\mathbf{c}_{1}^{T}, \ldots, \mathbf{c}_{G}^{T}\right]^{T}, \mathbf{c}_{g} \in\{0,1\}^{M_{g}}$, $g=1, \ldots, G$. By using the DMIM technique, the $g$ th subgroup of coded bits $\mathbf{c}_{g}$ is mapped to the $g$ th subframe $\mathbf{x}_{g} \triangleq$ $\left[x_{g}(1), \ldots, x_{g}\left(N_{g}\right)\right]^{T}$, which is comprised of $K_{g}$ symbols drawn from the $P_{A}$-ary constellation set $\dot{\mathcal{S}}=\left\{\dot{s}_{1}, \ldots, \dot{s}_{P_{A}}\right\}$ (denoted by "Mode A") and the remaining $\left(N_{g}-K_{g}\right)$ symbols from the $P_{B}$-ary constellation set $\ddot{\mathcal{S}}=\left\{\ddot{s}_{1}, \ldots, \ddot{s}_{P_{B}}\right\}$ (denoted by "Mode B") ${ }^{2}$. Specifically, the $M_{g}$ bits in $\mathbf{c}_{g}$ are further

\footnotetext{
${ }^{2}$ We tend to chose PSK instead of QAM in the two modes, due to the fact that PSK is naturally less sensitive to nonlinearities than QAM.
}

divided into smaller segments of $M_{g_{1}}, M_{g_{A}}$ and $M_{g_{B}}$ bits for different purpose: The first $M_{g_{\mathrm{I}}}=\left[\log _{2}\left(\begin{array}{c}N_{g} \\ K_{g}\end{array}\right)\right]$ bits enter the mode selector to separate the indices of $\mathbf{x}_{g}$ into two index subsets $\mathcal{I}_{g}^{A}=\left\{i_{g, 1}^{A}, \ldots, i_{g, K_{g}}^{A}\right\}$ and $\mathcal{I}_{g}^{B}=\left\{i_{g, 1}^{B}, \ldots, i_{g, N_{g}-K_{g}}^{B}\right.$, where $i_{g, m}^{A}, i_{g, l}^{B} \in\left\{1, \ldots, N_{g}\right\}$ for $m=1, \ldots, K_{g}$ and $l=1, \ldots, N_{g}-K_{g}$; the remaining $M_{g_{A}}=K_{g} \log _{2} P_{A}$ and $M_{g_{B}}=\left(N_{g}-K_{g}\right) \log _{2} P_{B}$ bits are mapped to $K_{g} P_{A}$-ary symbols $\mathbf{s}_{g}^{A}=\left[s_{g}^{A}(1), \ldots, s_{g}^{A}\left(K_{g}\right)\right]^{T} \in \dot{\mathcal{S}}^{K_{g}}$ and to $\left(N_{g}-\right.$ $\left.K_{g}\right) P_{B}$-ary symbols $\mathbf{s}_{g}^{B}=\left[s_{g}^{B}(1), \ldots, s_{g}^{B}\left(N_{g}-K_{g}\right)\right]^{T} \in$ $\ddot{\mathcal{S}}^{\left(N_{g}-K_{g}\right)}$, respectively. Given the mode permutation chosen by the mode selector, the symbols in $\mathbf{s}_{g}^{A}$ and $\mathbf{s}_{g}^{B}$ are then assigned to the indices in $\mathcal{I}_{g}^{A}$ and $\mathcal{I}_{g}^{B}$, respectively, yielding the $g$ th subframe $\mathbf{x}_{g}$. In other words, for $i=1, \ldots, N_{g}$, we have $x_{g}(i) \in \dot{\mathcal{S}}$ for $i \in \mathcal{I}_{g}^{A}$, and $x_{g}(i) \in \ddot{\mathcal{S}}$ for $i \in \mathcal{I}_{g}^{B}$. Note that with a little benign abuse of notation, we may denote the coded bits corresponding to the vector $\mathbf{x}_{g}$ as $\mathbf{c}_{g}=\left[c_{g, 1}^{\mathrm{IM}}, \ldots, c_{g, M_{g_{\mathrm{I}}}}^{\mathrm{IM}}, c_{g, 1}^{A}, \ldots, c_{g, M_{g_{A}}}^{A}, c_{g, 1}^{B}, \ldots, c_{g, M_{g_{B}}}^{B}\right]^{T}$, where $c_{g, i}^{\mathrm{IM}}$ is the $i$-th IM bit; $c_{g, \jmath}^{A}$ and $c_{g, k}^{B}$ are the $\jmath$-th and the $k$-th ordinary constellation mapping bits corresponding to the symbols belonging to $\dot{\mathcal{S}}$ and $\dot{\mathcal{S}}$, respectively.

Let us refer to $\mathcal{I}_{g}^{A}$ as the mode-selection pattern, since the subset $\mathcal{I}_{g}^{B}$ is determined once $\mathcal{I}_{g}^{A}$ is known. Naturally, there are $\left(\begin{array}{l}N_{g} \\ K_{g}\end{array}\right)$ possible permutations, but only $\Upsilon \triangleq 2^{M_{g_{\mathrm{I}}}}$ legitimate mode-selection patterns determined by the $M_{g_{\mathrm{I}}}$ IM bits are employed by the transmitter. Denote the predefined legitimate set as $\mathcal{I}^{A} \triangleq\left\{\mathcal{I}^{A}(1), \ldots, \mathcal{I}^{A}(\Upsilon)\right\}$. The fact that $\mathcal{I}_{g}^{A}$ should belong to $\mathcal{I}^{A}$ is referred to here as the mode-selection pattern constraint. Considering that existing mode mapping methods tend to introduce error propagation if different modulation orders are employed [17], [18], [21], we use the improved mapping rule provided in Table I for $N_{g}=4$ and $K_{g}=2$ as an example. The legitimate set of mode-selection patterns in this example is $\mathcal{I}^{A}=\{\{1,3\},\{1,4\},\{2,3\},\{2,4\}\}$. Specifically, according to the look-up Table I, if the $M_{g_{\mathrm{I}}}$ IM bits of the mode selector are [00], the first and third entries of $\mathbf{x}_{g}$ are $P_{A}$-ary symbols associated with the following $M_{g_{A}}$ bits, while the other two entries are $P_{B}$-ary symbols associated with the remaining $M_{g_{B}}$ bits. 
Finally, the frame buffer in Fig. 1 concatenates the $G$ subframes $\left\{\mathbf{x}_{g}\right\}_{g=1}^{G}$, creating the length- $N$ data frame $\mathbf{x}=$ $\left[x_{1}, \ldots, x_{N}\right]^{T}$. The effective transmission rate of the SatDMIM scheme is given by

$$
R=R_{c} \frac{\left\lfloor\log _{2}\left(\begin{array}{l}
N_{g} \\
K_{g}
\end{array}\right)\right\rfloor+K_{g} \log _{2} P_{A}+\left(N_{g}-K_{g}\right) \log _{2} P_{B}}{N_{g}} .
$$

\section{B. Dispersive Nonlinear Satellite Channels}

As shown in Fig. 1, the satellite transponder consists of an input demultiplexer (IMUX) filter, a high power amplifier (HPA) and an output multiplexer (OMUX) filter [38]. For reasons of power efficiency, the HPA always operates close to its saturation region, which inevitably introduces nonlinear memoryless distortion [28]. The IMUX and OMUX filter$\mathrm{s}$ placed before and after HPA are used for reducing the effect of adjacent channels and for mitigating the spectral broadening caused by the nonlinear amplifier, respectively. However, the employment of these two filters may introduce ISI. We assume that the characteristics of both the HPA, as well as the IMUX and OMUX filters are perfectly known at both the transmitter and receiver sides. In order to describe the resultant effect of nonlinearities with memory, numerous models have been developed in the literature, including the polynomial model [39], as well as the Hammerstein model [40] and the Volterra model [34]. The Volterra model, derived by decomposing the nonlinear channels using the Taylor series, has a generic expression. Based on the Volterra model, the dispersive nonlinear satellite channels can be modelled by a finite state machine, which has been widely applied for the receiver design in classical Satcom [27], [29], [30], [41]. Note that considering several high-order Volterra series terms may be able to describe the actual satellite channels more accurately and help design improved nonlinear detectors. However, the resultant modelling complexity can become excessive and the instability of the nonlinearity compensation tends to increase [42]. Hence, we use the popular third-order Volterra-series expansion model (refer to [34] for details of nonlinear satellite channels) to decompose the $n$th discrete received symbol as:

$$
r_{n}=x_{n} h_{0}+\underbrace{\sum_{l=1}^{L} x_{n-l} h_{l}}_{\text {Linear ISI }}+\underbrace{\sum_{i=0}^{L} \sum_{j \geq i}^{L} \sum_{k=0}^{L} x_{n-i} x_{n-j} x_{n-k}^{*} h_{i j k}}_{\text {Nonlinear ISI }}+w
$$

where $L$ represents the dispersion distortion of the nonlinear channels in terms of the number of symbols; $h_{l}$ and $h_{i j k}$ are the first- and third-order Volterra kernels ${ }^{3}$, respectively; and $w_{n}$ is the zero-mean complex circularly symmetric Gaussian noise sample with a variance of $2 \sigma^{2}$. In (1), $\sum_{l=1}^{L} x_{n-l} h_{l}$ and $\sum_{i=1}^{L} \sum_{j \geq i}^{L} \sum_{k=1}^{L} x_{n-i} x_{n-j} x_{n-k}^{*} h_{i j k}$ are the linear-distortion-induced ISI and the nonlinear-distortioninduced ISI, respectively.

\footnotetext{
${ }^{3}$ The absence of even-order terms is due to the fact that they generate spectral components which lie outside the channel bandwidth [34].
}

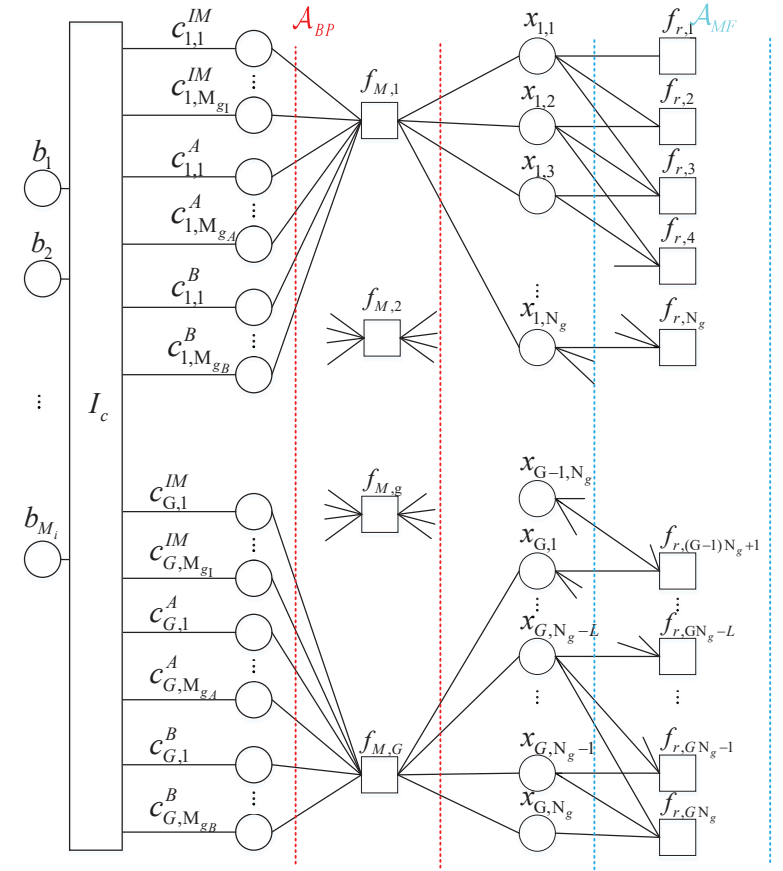

Fig. 2. Factor graph representation of the probabilistic model (3).

\section{Receiver}

In contrast to the conventional SC scheme, the symbols in Sat-DMIM become correlated due to the mode-selection mapping in the time domain. In this case, the a priori information of each Sat-DMIM symbol gleaned from the SISO decoder is subject to the mode-selection pattern constraint. As a further aggravation, if not fully compensated, the dispersive nonlinear satellite channels may significantly degrade the detection accuracy of both the mode-selection mapping symbols and the APSK/QAM symbols. Therefore, the receiver has to jointly detect the IM bits and ordinary constellation mapping bits of LDPC-coded Sat-DMIM, while coping with both linear and nonlinear ISI. As illustrated in Fig. 1, by exploiting the turbo-equalisation principle [43], the SISO demodulator exploits the structured a priori information provided by the SISO decoder from the previous iteration to decouple the Sat-DMIM symbols. Given the estimates of the Sat-DMIM symbols provided by the SISO demodulator, the demappers of the mode selector, as well as of the Mode A and Mode B, update the a posteriori log-likelihood ratios (LLRs) of LDPCcoded bits within each subframe. By extracting the a priori information from the a posteriori probability distributions, the SISO decoder obtains the so-called extrinsic LLRs and then uses them to perform Bahl, Cocke, Jelinek and Raviv (BCJR) decoding [44] for the LDPC-coded frame. The output of the SISO decoder is then fed back to the SISO demodulator for the next iteration, as seen in Fig. 1.

\section{Amalgamated BP-MF-BASEd Iterative DETECTION ALGORITHM}

\section{A. Probabilistic Representation and Factor Graph}

The goal of the optimal receiver is to find the estimates of information bits in $\mathbf{b}$ based on the maximum a posteriori 


$$
\begin{aligned}
& f_{r, n}\left(x_{n}, \ldots, x_{n-L}, r_{n}\right) \propto \exp \left\{-\frac{1}{2 \sigma^{2}}\left|r_{n}-\sum_{l=0}^{L} x_{n-l} h_{l}-\sum_{i=0}^{L} \sum_{\jmath \geq i}^{L} \sum_{k=0}^{L} x_{n-i} x_{n-\jmath} x_{n-k}^{*} h_{i \jmath k}\right|^{2}\right\} \\
& \propto \exp \left\{-\frac{1}{2 \sigma^{2}}\left\{\left|x_{n}\right|^{6}\left|h_{000}\right|^{2}+\left|x_{n}\right|^{4}\left[\psi_{n, n}^{o \triangleleft o, 2}+\psi_{n, n}^{o o \triangleleft, 2}+2 \Re\left[h_{000}^{*}\left(h_{0}+\psi_{n, n}^{o \triangleleft \triangleleft}\right)\right]\right]\right.\right. \\
& +\left|x_{n}\right|^{2}\left[\left|h_{0}\right|^{2}+\psi_{n, n}^{o \triangleleft \triangleleft, 2}+\psi_{n, n}^{\triangleleft \triangleleft o, 2}+2 \Re\left[\left(-r_{n}+\varepsilon_{n, n}\right)^{*} \psi_{n, n}^{o \triangleleft o}+h_{0}^{*} \psi_{n, n}^{o \triangleleft \triangleleft}\right]\right]+\left|\varepsilon_{n, n}\right|^{2} \\
& +2 \Re\left[\left|x_{n}\right|^{4} x_{n}\left[h_{000}\left(\psi_{n, n}^{o \triangleleft o}\right)^{*}+h_{000}^{*} \psi_{n, n}^{o o \triangleleft}\right]+\left|x_{n}\right|^{2} x_{n}^{2}\left[h_{000}\left(\psi_{n, n}^{\triangleleft \triangleleft o}\right)^{*}+\left(\psi_{n, n}^{o \triangleleft o}\right)^{*} \psi_{n, n}^{o o \triangleleft}\right]+x_{n}^{3} \psi_{n, n}^{o o \triangleleft}\left(\psi_{n, n}^{\triangleleft \triangleleft o}\right)^{*}\right. \\
& +\left|x_{n}\right|^{2} x_{n}\left[\left(-r_{n}+\varepsilon_{n, n}\right)^{*} h_{000}+\left(\psi_{n, n}^{o \triangleleft o}\right)^{*}\left(h_{0}+\psi_{n, n}^{o \triangleleft \triangleleft}\right)+\psi_{n, n}^{o o \triangleleft}\left(h_{0}+\psi_{n, n}^{o \triangleleft \triangleleft}\right)^{*}+\psi_{n, n}^{o \triangleleft o}\left(\psi_{n, n}^{\triangleleft \triangleleft o}\right)^{*}\right]-r_{n}^{*} \varepsilon_{n, n} \\
& \left.\left.\left.+x_{n}^{2}\left[\left(-r_{n}+\varepsilon_{n, n}\right)^{*} \psi_{n, n}^{o o \triangleleft}+\left(h_{0}+\psi_{n, n}^{o \triangleleft \triangleleft}\right)\left(\psi_{n, n}^{\triangleleft \triangleleft o}\right)^{*}\right]+x_{n}\left[\left(-r_{n}+\varepsilon_{n, n}\right)^{*}\left(h_{0}+\psi_{n, n}^{o \triangleleft \triangleleft}\right)+\left(-r_{n}+\varepsilon_{n, n}\right)\left(\psi_{n, n}^{\triangleleft \triangleleft o}\right)^{*}\right]\right]\right\}\right\} \text {, } \\
& \psi_{n, n}^{o \triangleleft o}=\sum_{i=1}^{L} x_{n-i} h_{0 i 0}, \quad \psi_{n, n}^{o o \triangleleft}=\sum_{i=1}^{L} x_{n-i}^{*} h_{00 i}, \quad \psi_{n, n}^{o \triangleleft \triangleleft}=\sum_{i=1}^{L} \sum_{\jmath=1}^{L} x_{n-i} x_{n-\jmath}^{*} h_{0 i \jmath}, \quad \psi_{n, n}^{\triangleleft \triangleleft o}=\sum_{i=1}^{L} \sum_{\jmath \geq i}^{L} x_{n-i} x_{n-\jmath} h_{i \jmath 0}, \\
& \psi_{n, n}^{o \triangleleft o, 2}=\sum_{i=1}^{L}\left|x_{n-i}\right|^{2}\left|h_{0 i 0}\right|^{2}, \psi_{n, n}^{o o \triangleleft, 2}=\sum_{i=1}^{L}\left|x_{n-i}^{*}\right|^{2}\left|h_{00 i}\right|^{2}, \psi_{n, n}^{o \triangleleft \triangleleft, 2}=\sum_{i=1}^{L} \sum_{\jmath=1}^{L}\left|x_{n-i}\right|^{2}\left|x_{n-\jmath}^{*}\right|^{2}\left|h_{0 i \jmath}\right|^{2}, \psi_{n, n}^{\triangleleft \triangleleft o, 2}=\sum_{i=1}^{L} \sum_{\jmath \geq i}^{L}\left|x_{n-i}\right|^{2}\left|x_{n-\jmath}\right|^{2}\left|h_{i \jmath 0}\right|^{2}, \\
& \varepsilon_{n, n}=\sum_{i=1}^{L} x_{n-i} h_{i}+\sum_{i=1}^{L} \sum_{\jmath \geq i}^{L} \sum_{k=1}^{L} x_{n-i} x_{n-\jmath} x_{n-k}^{*} h_{i \jmath k} .
\end{aligned}
$$

(MAP) criterion:

$$
\begin{aligned}
\hat{b}_{i}^{\mathbf{M A P}} & =\arg \max _{b_{i}} p\left(b_{i} \mid \mathbf{r}\right) \\
& =\arg \max _{b_{i}} \sum_{\mathbf{b} \backslash b_{i}} p(\mathbf{b} \mid \mathbf{r}), \quad i=1, \ldots, M_{i},
\end{aligned}
$$

where $\mathbf{r} \triangleq\left[r_{1}, \ldots, r_{N}\right]^{T}$ denotes the sequence of received signal samples. As can be readily seen that the evaluation of (2) has an exponentially increasing complexity vs. the size $M_{i}$. To mitigate this excessive complexity, based on the received signal model in (1), we propose to factorize the joint a posteriori pdf of the information bits $\mathbf{b}$, the coded bits $\mathbf{c}$ and the Sat-DMIM symbols $\mathbf{x}$ as follows:

$$
\begin{aligned}
p(\mathbf{b}, \mathbf{c}, \mathbf{x} \mid \mathbf{r}) \propto p(\mathbf{r} \mid \mathbf{x}) p(\mathbf{x} \mid \mathbf{c}) p(\mathbf{c} \mid \mathbf{b}) p(\mathbf{b}) & \\
\propto & \mathbb{I}_{c}(\mathbf{c}, \mathbf{b}) \prod_{g=1}^{G} f_{M, g}\left(\mathbf{x}_{g} \mid \mathbf{c}_{g}\right) P(\mathbf{x}) \\
& \cdot \prod_{n=1}^{N} f_{r, n}\left(x_{n}, x_{n-1}, \ldots, x_{n-L}, r_{n}\right),
\end{aligned}
$$

where the indicator function $\mathbb{I}_{c}(\cdot)$ stands for the coding constraint and $f_{M, g}(\cdot)$ represents the mode-selection and PSK/QAM modulation constraints. In (3), the likelihood function $f_{r, n}(\cdot)$ is given by (5), where the variables $\psi_{n, n}^{o \triangleleft o}, \psi_{n, n}^{o o \triangleleft}$, $\psi_{n, n}^{o \triangleleft \triangleleft}, \psi_{n, n}^{\triangleleft \triangleleft o}, \psi_{n, n}^{o \triangleleft o, 2}, \psi_{n, n}^{o o \triangleleft, 2}, \psi_{n, n}^{o \triangleleft \triangleleft, 2}, \psi_{n, n}^{\triangleleft \triangleleft o, 2}$ and $\varepsilon_{n, n}$ that appear in (5) are given by (6). We observe that these variables are composed of the Sat-DMIM symbols $\left\{x_{n-1}, \ldots, x_{n-L}\right\}$, but they are unrelated to $x_{n}$, as a result of classifying the linear and nonlinear ISI terms in (1) with reference to $x_{n}$. If another variable $x_{m}, m=n-1, \ldots, n-L$, is the desired symbol, the function $f_{r, n}(\cdot)$ has to be rewritten by rearranging the ISI terms, which will lead to an expression similar to (5). Note that the sufficient statistics of $x_{m}, m=n, \ldots, n-L$, involved in the likelihood function $f_{r_{n}}(\cdot)$ are determined by the specific order of the Volterra model and are not dependent on the dispersion $L$. For example, there are a total of $\mathcal{L}^{2}$ fixed sufficient statistics of $x_{m}$, including $\left|x_{m}\right|^{6},\left|x_{m}\right|^{4},\left|x_{m}\right|^{2}$, $\Re\left\{\left|x_{m}\right|^{4} x_{m}\right\}, \Re\left\{\left|x_{m}\right|^{2} x_{m}^{2}\right\}, \Re\left\{x_{m}^{3}\right\}, \Re\left\{\left|x_{m}\right|^{2} x_{m}\right\}, \Re\left\{x_{m}^{2}\right\}$ and $\Re\left\{x_{m}\right\}$, where $\mathcal{L}$ is Volterra model order. The factor graph representation corresponding to the factorization in (3) is shown in Fig. 2 for the case of $L=2$.

As seen in Fig. 2, the Sat-DMIM symbols are correlated due to the mode-selection pattern constraint and owing to the memory in the overall satellite transponder response. The application of the standard sum-product algorithm [32] at both the constraint nodes $\left\{f_{M, g}\right\}_{g=1}^{G}$ and the observation nodes $\left\{f_{r, n}\right\}_{n=1}^{N}$ leads to a near-optimal forward-backward (FB) equalizer [28]. However, the implementation complexity of the FB equalizer is on the order of $\mathcal{O}\left[\left(P_{A}+P_{B}\right)^{L}\right]$, which may be deemed excessive for large constellations transmitted over nonlinear channels having long memory. To this end, we propose a low-complexity message passing algorithm by classifying the factors in Fig. 2 into two regions, i.e. the MF-region $\mathcal{A}_{\mathrm{MF}}=\left\{f_{r, n} ; n \in[1: N]\right\}$ and the BP-region $\mathcal{A}_{\mathrm{BP}}=\left\{f_{M, g} ; g \in[1: G]\right\}$. This is motivated by the observation that the MF method is competitive with the exponential distribution family, whilst the BP algorithm delivers remarkable performance for discrete probabilistic models [45].

\section{B. Message Passing at the Constraint Nodes}

Instead of directly treating the correlated Sat-DMIM symbols as independent Gaussian variables, we explicitly construct the a priori information of Sat-DMIM symbols by taking full account of the mode-selection pattern constraint. Let us denote the extrinsic probabilities of coded bits provided by the SISO decoder as $\left\{P\left(c_{g, 1}^{\mathrm{IM}}\right), \ldots, P\left(c_{g, M_{g_{\mathrm{I}}}}^{\mathrm{IM}}\right), P\left(c_{g, 1}^{A}\right), \ldots, P\left(c_{g, M_{g_{A}}}^{A}\right), P\left(c_{g, 1}^{B}\right), \ldots\right.$, $\left.P\left(c_{g, M_{g_{B}}}^{B}\right)\right\}$. Then the a priori probabilities of the legitimate 


$$
\begin{aligned}
& D\left(x_{i}=\dot{s}_{m}\right) \propto \sum_{\gamma: i \in \mathcal{I}^{A}(\gamma)} P\left(\mathcal{I}_{g}^{A}=\mathcal{I}^{A}(\gamma)\right) \mu_{x_{i} \rightarrow f_{M, g}}\left(x_{i}\right) P\left(x_{i}=\dot{s}_{m}\right) \\
& \cdot \prod_{\jmath \in \mathcal{I}^{A}(\gamma), \jmath \neq i}\left(\sum_{\dot{s} \in \dot{\mathcal{S}}} \mu_{x_{\jmath} \rightarrow f_{M, g}}\left(x_{\jmath}\right) P\left(x_{\jmath}=\dot{s}\right)\right) \prod_{k \in \overline{\mathcal{I}}^{A}(\gamma)}\left(\sum_{\ddot{s} \in \ddot{\mathcal{S}}} \mu_{x_{k} \rightarrow f_{M, g}}\left(x_{k}\right) P\left(x_{k}=\ddot{s}\right)\right), \dot{s}_{m} \in \dot{\mathcal{S}}, m=1, \ldots, P_{A} . \\
& E\left(x_{i}=\ddot{s}_{l}\right) \propto \sum_{\gamma: i \in \overline{\mathcal{I}}^{A}(\gamma)} P\left(\mathcal{I}_{g}^{A}=\overline{\mathcal{I}}^{A}(\gamma)\right) \mu_{x_{i} \rightarrow f_{M, g}}\left(x_{i}\right) P\left(x_{i}=\ddot{s}_{l}\right) \\
& \cdot \prod_{\jmath \in \overline{\mathcal{I}}^{A}(\gamma), \jmath \neq i}\left(\sum_{\ddot{s} \in \ddot{\mathcal{S}}} \mu_{x_{\jmath} \rightarrow f_{M, g}}\left(x_{\jmath}\right) P\left(x_{\jmath}=\ddot{s}\right)\right) \prod_{k \in \mathcal{I}^{A}(\gamma)}\left(\sum_{\dot{s} \in \dot{\mathcal{S}}} \mu_{x_{k} \rightarrow f_{M, g}}\left(x_{k}\right) P\left(x_{k}=\dot{s}\right)\right), \ddot{s}_{l} \in \ddot{\mathcal{S}}, l=1, \ldots, P_{B} .
\end{aligned}
$$

pattern and the ordinary APSK/QAM symbols are given by

$$
\begin{aligned}
& P\left(\mathcal{I}_{g}^{A}=\mathcal{I}^{A}(\gamma)\right)= \prod_{i=1}^{M_{g_{\mathrm{I}}}} P\left(c_{g, i}^{\mathrm{IM}}=c_{i}^{\gamma}\right), \\
& \gamma=1, \ldots, \Upsilon \\
& P\left(x_{g}(\jmath)=\dot{s}_{m}\right)= \prod_{i=1}^{\log _{2} P_{A}} P\left(c_{g,(\jmath-1) \log _{2} P_{A}+i}^{A}=c_{i}^{\dot{s}_{m}}\right), \\
& P\left(x_{g}(k)=\ddot{s}_{l}\right)= \prod_{i=1}^{\log _{2} P_{B}} P\left(c_{g,(k-1) \operatorname{Iog}_{2} P_{B}+i}^{B}(\gamma), m=1, \ldots, c_{i}^{\ddot{s}_{l}}\right), \\
& k \in \overline{\mathcal{I}}^{A}(\gamma), l=1, \ldots, P_{B},
\end{aligned}
$$

where the sequences of coded bits $\left[c_{1}^{\gamma}, \ldots, c_{M_{g_{\mathrm{I}}}}^{\gamma}\right]^{T}$, $\left[c_{1}^{\dot{s}_{m}}, \ldots, c_{\log _{2} P_{A}}^{\dot{s}_{m}}\right]^{T}$ and $\left[c_{1}^{\ddot{s}_{l}}, \ldots, c_{\log _{2} P_{B}}^{\ddot{s}_{l}}\right]^{T} \quad$ correspond to the $\gamma$ th legitimate pattern $\mathcal{I}^{A}(\gamma)$, to the $m$ th symbol $\dot{s}_{m}$ belonging to mode $\mathrm{A}$ and to the $l$ th symbol $\ddot{s}_{l}$ belonging to mode $\mathrm{B}$, respectively. According to the process of modeselection mapping as described in Section II, the a priori probabilities of the Sat-DMIM symbols can be constructed by

$$
\begin{aligned}
P(\mathbf{x}) & =\prod_{g=1}^{G} P_{\mathbf{x}_{g}}\left(\mathbf{x}_{g}\right), \\
P_{\mathbf{x}_{g}}\left(\mathbf{x}_{g}\right) & =\sum_{\gamma=1}^{\Upsilon} P\left(\mathcal{I}_{g}^{A}=\mathcal{I}^{A}(\gamma)\right) \prod_{i \in \mathcal{I}^{A}(\gamma)}\left(\sum_{\dot{s} \in \dot{\mathcal{S}}} \delta\left(x_{i}-\dot{s}\right) P\left(x_{i}\right)\right) \\
\cdot & \prod_{\jmath \in \overline{\mathcal{I}}^{A}(\gamma)}\left(\sum_{\ddot{s} \in \ddot{\mathcal{S}}} \delta\left(x_{\jmath}-\ddot{s}\right) P\left(x_{\jmath}\right)\right),
\end{aligned}
$$

where $i, \jmath \in I_{g} \triangleq\left\{(g-1) N_{g}+1: g N_{g}\right\}$ and $\overline{\mathcal{I}}^{A}(\gamma)$ is the complementary set of $\mathcal{I}^{A}(\gamma)$. Given (8) and the message $\mu_{x_{i} \rightarrow f_{M, g}}\left(x_{i}\right)$ which will be later updated according to (20), the a posteriori pdf $b\left(x_{i}\right)$ of the Sat-DMIM symbol $x_{i}$ can be formulated as

$$
b\left(x_{i}=x\right)=\left\{\begin{array}{l}
\frac{D\left(x_{i}=x\right)}{\frac{\sum_{\dot{s} \in \dot{\mathcal{S}}} D\left(x_{i}=\dot{s}\right)+\sum_{\ddot{s} \in \ddot{\mathcal{S}}} E\left(x_{i}=\ddot{s}\right)}{E\left(x_{i}=x\right)}, x \in \mathcal{S},} \\
\frac{\sum_{\dot{s} \in \dot{\mathcal{S}}} D\left(x_{i}=\dot{s}\right)+\sum_{\ddot{s} \in \mathcal{S}} E\left(x_{i}=\ddot{s}\right)}{x}, x \in \ddot{\mathcal{S}},
\end{array}\right.
$$

where $D(\cdot)$ and $E(\cdot)$ are given by (10) and (11), respectively. Then the a posteriori mean and variance of $x_{i}$ are calculated
Note that for the first iteration no extrinsic information arrives from the SISO decoder. By appropriately designing the legitimate pattern set, the structured a priori pdf of $\mathbf{x}_{g}$ can be directly written as

$$
\begin{aligned}
P_{\mathbf{x}_{g}}^{0}\left(\mathbf{x}_{g}\right)=\frac{1}{\Upsilon} \sum_{\gamma=1}^{\Upsilon} & {\left[\prod_{i \in \mathcal{I}^{A}(\gamma)}\left(\frac{1}{P_{A}} \sum_{\dot{s} \in \dot{\mathcal{S}}} \delta\left(x_{i}-\dot{s}\right)\right)\right.} \\
\cdot & \left.\prod_{\jmath \in \overline{\mathcal{I}}^{A}(\gamma)}\left(\frac{1}{P_{B}} \sum_{\ddot{s} \in \ddot{\mathcal{S}}} \delta\left(x_{\jmath}-\ddot{s}\right)\right)\right] .
\end{aligned}
$$

by

$$
\begin{aligned}
\hat{x}_{i} & =\sum_{\dot{s} \in \dot{\mathcal{S}}} \dot{s} b\left(x_{i}=\dot{s}\right)+\sum_{\ddot{s} \in \mathcal{S}} \ddot{s} b\left(x_{i}=\ddot{s}\right), \\
v_{x_{i}} & =\sum_{\dot{s} \in \dot{\mathcal{S}}}|\dot{s}|^{2} b\left(x_{i}=\dot{s}\right)+\sum_{\ddot{s} \in \mathcal{S}}|\ddot{s}|^{2} b\left(x_{i}=\ddot{s}\right)-\left|\hat{x}_{i}\right|^{2} .
\end{aligned}
$$

Accordingly, the initial a priori mean and variance of $x_{i}$ are given by

$$
\begin{aligned}
\hat{x}_{i}^{0} & =\sum_{\dot{s} \in \dot{\mathcal{S}}} \dot{s} \frac{K_{g}}{P_{A} N_{g}}+\sum_{\ddot{s} \in \ddot{\mathcal{S}}} \ddot{s} \frac{N_{g}-K_{g}}{P_{B} N_{g}} \\
v_{x_{i}}^{0} & =\sum_{\dot{s} \in \dot{\mathcal{S}}}|\dot{s}|^{2} \frac{K_{g}}{P_{A} N_{g}}+\sum_{\ddot{s} \in \ddot{\mathcal{S}}}|\ddot{s}|^{2} \frac{N_{g}-K_{g}}{P_{B} N_{g}}-\left|\hat{x}_{i}^{0}\right|^{2} .
\end{aligned}
$$

\section{Message Passing at the Observation Nodes}

According to the MF rule (refer to [45] for MF rules), we can calculate the message $\mu_{f_{r, m} \rightarrow x_{n}}\left(x_{n}\right)$ forwarded from the observation node $f_{r, m}$ to the Sat-DMIM symbol variable node $x_{n}, n \in[m, m-L]$, by

$$
\begin{aligned}
& \mu_{f_{r, m} \rightarrow x_{n}}\left(x_{n}\right) \\
& \propto \exp \left\{E_{\prod_{m \leq i \leq m-L, i \neq n} b\left(x_{i}\right)} \log f_{r, m}\left(x_{m}, \ldots, x_{m-L}, r_{m}\right)\right\} \\
& \triangleq \exp \left\{-\frac{1}{2 \sigma^{2}}\left(\hat{\varphi}_{r_{m}, n}^{3,3}\left|x_{n}\right|^{6}+\hat{\varphi}_{r_{m}, n}^{2,2}\left|x_{n}\right|^{4}+\hat{\varphi}_{r_{m}, n}^{1,1}\left|x_{n}\right|^{2}\right.\right. \\
& \quad+2 \Re\left\{\hat{\phi}_{r_{m}, n}^{3,2}\left|x_{n}\right|^{4} x_{n}+\hat{\phi}_{r_{m}, n}^{3,1}\left|x_{n}\right|^{2} x_{n}^{2}+\hat{\phi}_{r_{m}, n}^{3,0} x_{n}^{3}\right. \\
& \left.\left.\left.\quad+\hat{\phi}_{r_{m}, n}^{2,1}\left|x_{n}\right|^{2} x_{n}+\hat{\phi}_{r_{m}, n}^{2,0} x_{n}^{2}+\hat{\phi}_{r_{m}, n}^{1,0} x_{n}\right\}\right)\right\}
\end{aligned}
$$

$$
\triangleq \exp \left\{-\frac{1}{2 \sigma^{2}}\left(\sum_{q, v} \hat{\varphi}_{r_{m}, n}^{q, v} x_{n}^{q}\left(x_{n}^{*}\right)^{v}+2 \Re\left\{\sum_{k, l} \hat{\phi}_{r_{m}, n}^{k, l} x_{n}^{k}\left(x_{n}^{*}\right)^{l}\right\}\right)\right\}
$$




$$
\begin{aligned}
\hat{\varphi}_{r_{n}, n}^{2,2} & =E_{\prod_{n \leq i \leq n-L, i \neq n} b\left(x_{i}\right)}\left[\psi_{n, n}^{o \triangleleft o, 2}+\psi_{n, n}^{o o \triangleleft, 2}+2 \Re\left[h_{000}^{*}\left(h_{0}+\psi_{n, n}^{o \triangleleft \triangleleft}\right)\right]\right], \\
& =\underbrace{\sum_{i=1}^{L}\left(\left|\hat{x}_{n-i}\right|^{2}+v_{x_{n-i}}\right)\left|h_{0 i 0}\right|^{2}}_{\hat{\psi}_{n}^{O \triangleleft o, 2}}+\underbrace{\sum_{i=1}^{L}\left(\left|\hat{x}_{n-i}\right|^{2}+v_{x_{n-i}}\right)\left|h_{00 i}\right|^{2}}_{\hat{\psi}_{n}^{o \circlearrowleft \triangleleft, 2}}+2 \Re\left[h_{000}^{*}(h_{0}+\underbrace{\left.\left.\sum_{i=1}^{L} \sum_{j=1}^{L} \hat{x}_{n-i} \hat{x}_{n-j}^{*} h_{0 i j}\right)\right]}_{\hat{\psi}_{n}^{O \triangleleft}},\right.
\end{aligned}
$$

where the a posteriori pdf $b\left(x_{i}\right)$ of $x_{i}$ is updated in the previous iteration using (9); the parameters $\hat{\varphi}_{r_{m}, n}^{q, v}$ and $\hat{\phi}_{r_{m}, n}^{k, l}$ consist of the products of the Volterra kernels and of the first- as well as of the second-order moments of the variables $\left\{x_{m}, \ldots, x_{m-L}\right\}$ except for $x_{n}$; and the parameters $\{q, v\}$ and $\{k, l\}$ indicate the number of variables $x_{n}$ and $x_{n}^{*}$ involved in the sufficient statistics that are associated with the parameters $\hat{\varphi}_{r_{m}, n}^{q, v}$ and $\hat{\phi}_{r_{m}, n}^{k, l}$. Considering that the Sat-DMIM symbols are discrete variables, the parameters $\hat{\varphi}_{r_{m}, n}^{q, v}$ and $\hat{\phi}_{r_{m}, n}^{k, l}$ can be calculated by using the enumeration method of [46]. For example, when we have $m=n$, the parameter associated with $\left|x_{n}\right|^{4}$, namely, $\hat{\varphi}_{r_{n}, n}^{2,2}$ can be calculated by (18), where the variables $\psi_{n, n}^{o \triangleleft o, 2}, \psi_{n, n}^{o \circ \triangleleft, 2}$ and $\psi_{n, n}^{o \triangleleft \triangleleft}$ that appear in (17) are given by (6); the a posteriori mean and variance of $x_{i}$ are calculated by (12). The other parameters can be updated in a similar way.

Given the message $\mu_{f_{r, m} \rightarrow x_{n}}\left(x_{n}\right), m=n, \ldots, n+L$, the message forwarded from the variable node (VN) $x_{n}$ to the constraint node $(\mathrm{CN}) f_{M, g}$ can then be updated by

$$
\begin{aligned}
& \mu_{x_{n} \rightarrow f_{M, g}}\left(x_{n}\right) \propto \prod_{m=n}^{n+L} \mu_{f_{r, m} \rightarrow x_{n}}\left(x_{n}\right) \\
& \propto \exp \left\{-\frac{1}{2 \sigma^{2}} \sum_{m=n}^{n+L}\left(\sum_{q, v} \hat{\varphi}_{r_{m}, n}^{q, v} x_{n}^{q}\left(x_{n}^{*}\right)^{v}\right.\right. \\
& \left.\left.+2 \Re\left\{\sum_{k, l} \hat{\phi}_{r_{m}, n}^{k, l} x_{n}^{k}\left(x_{n}^{*}\right)^{l}\right\}\right)\right\},
\end{aligned}
$$

where $n \in I_{g}$. It is readily seen that updating the messages $\mu_{f_{r, m} \rightarrow x_{n}}\left(x_{n}\right), m=n, \ldots, n+L$, and $\mu_{x_{n} \rightarrow f_{M, g}}\left(x_{n}\right)$, $n=1, \ldots, N$, only requires updating their parameters by calculating the products of specific combinations of the firstand second-order moments of $L$ consecutive variables within each Sat-DMIM frame.

By substituting the message $\mu_{x_{n} \rightarrow f_{M, g}}\left(x_{n}\right)$ into (10) and (11), the belief of $x_{n}$ can be immediately updated according to (9). Meanwhile, we obtain the belief of $\mathbf{x}_{g}$ by

$$
b\left(\mathbf{x}_{g}\right)=P_{\mathbf{x}_{g}}\left(\mathbf{x}_{g}\right) \prod_{i \in I_{g}} \mu_{x_{i} \rightarrow f_{M, g}}\left(x_{i}\right) .
$$

Then the extrinsic LLRs of the $i$-th mode-selection mapping bit $c_{g, i}^{\mathrm{IM}}$, the $j$ th Mode A mapping bit $c_{g, j}^{A}$ and the $k$-th Mode B mapping bit $c_{g, k}^{B}$ associated with the $g$-th Sat-DMIM symbol vector $\mathbf{x}_{g}$, i.e., $L^{\operatorname{extr}}\left(c_{g, i}^{\mathrm{IM}}\right), L^{\operatorname{extr}}\left(c_{g, j}^{A}\right)$ and $L^{\operatorname{extr}}\left(c_{g, k}^{B}\right)$, respectively, can be iteratively updated based on the turboprinciple [43].

\section{Message Passing Scheduling}

The message passing schedule for the proposed joint SatDMIM symbol detection and decoding algorithm based on the amalgamated BP-MF method in nonlinear dispersive satellite channels, which is referred to as BP-MF, is summarized in Algorithm 1.

Algorithm 1 The Proposed Joint Sat-DMIM Symbols Detection and Decoding Algorithm Based on the Amalgamated BPMF Method in Nonlinear Dispersive Satellite Channels.

1: Initialization: $t=0$ (iteration index)

2: For $n=1, \ldots, N$, compute $\hat{x}_{n}^{0}$ and $v_{x_{n}}^{0}$ according to (14).

3: Set $t=1$ and repeat the following steps until $\sum_{n} \mid \hat{x}_{n}^{t+1}-$ $\left.\hat{x}_{n}^{t}\right|^{2}<\epsilon \sum_{n}\left|\hat{x}_{n}^{t}\right|^{2}$ or $t>T_{\operatorname{ex}(\max )}$, where $\epsilon$ is a prespecified error tolerance and $T_{\operatorname{ex}(\max )}$ is the maximum number of iterations.

4: - Decoupling step: For $n=1, \ldots, N, m=n, \ldots, n+L$, compute the message $\mu_{f_{r, m} \rightarrow x_{n}}\left(x_{n}\right)$ and $\mu_{x_{n} \rightarrow f_{M, g}}\left(x_{n}\right)$ from (16) and (20), respectively.

5: - Decoding step: For $g=1 \ldots, G, i=1, \ldots, M_{g_{\mathrm{I}}}, j=$ $1, \ldots, M_{g_{A}}$ and $k=1, \ldots, M_{g_{B}}$, compute the extrinsic LLRs $L^{\text {extr }}\left(c_{g, i}^{\mathrm{IM}}\right), L^{\operatorname{extr}}\left(c_{g, j}^{A}\right)$ and $L^{\operatorname{extr}}\left(c_{g, k}^{B}\right)$, and then forward them to the SISO decoder to perform the standard BCJR decoding [44].

6: Compute (7) and (8) by using the extrinsic information emerging from the SISO decoder.

7: - Denoising step: For $n=1, \ldots, N$, compute $\left\{\hat{x}_{n}^{t}, v_{x_{n}}^{t}\right\}$ from (12).

\section{PARTial Linearization Approximation-Based ITERATIVE Detection Algorithm}

\section{A. Complexity Reduction Using the Simplification of Message Parameters}

Observe by referring to (6), (16) and (20), that on the order of $\mathcal{O}\left[(L+1)\left(\frac{1}{2} L^{3}+\frac{7}{2} L^{2}+6 L\right)\right]$ multiplications are involved in detecting each Sat-DMIM symbol. This means that the computational complexity of the amalgamated BPMF algorithm is still considerable for a long channel memory, since it grows with the fourth power of the channel's dispersion. Therefore, a reduced-complexity iterative detection algorithm is required for simplifying the message updating process of $\mu_{f_{r, m} \rightarrow x_{n}}\left(x_{n}\right)$ and $\mu_{x_{n} \rightarrow f_{M, g}}\left(x_{n}\right)$ from (16) and (20), respectively. Due to the very similar forms of messages $\left\{\mu_{f_{r, m} \rightarrow x_{n}}\left(x_{n}\right)\right\}_{m=n}^{n+L}$, in the following, we will focus our attention the approximation of the message $\mu_{f_{r, n} \rightarrow x_{n}}\left(x_{n}\right)$, noting that similar results hold for other messages. Considering that these messages all have compact parametric 
representations, our proposed low-complexity message passing method reduces to approximating and propagating the function parameters. Firstly, the estimates of variable $\varepsilon_{n, n}$ in (6) is approximated as

$$
\hat{\varepsilon}_{n, n} \approx \underline{\hat{\varepsilon}}_{n, n} \triangleq \sum_{i=1}^{L} \hat{x}_{n-i} h_{i},
$$

assuming $h_{i \jmath k}=0$ for $i, \jmath, k \neq 0$. This operation is equivalent to discarding some high-order terms in the message $\mu_{f_{r, n} \rightarrow x_{n}}\left(x_{n}\right)$. Note that the approximation in (22) is different from the nonlinear soft interference canceler of [26], which simply neglects the contribution of third-order Volterra terms involved in the received signal. Instead, we make approximations for updating complex messages by simplifying their parameters. In our proposed approximation method, not all $h_{i j k}$, $i, \jmath, k \neq 0$, are treated as zeros when calculating (20), because these Volterra kernels are involved in calculating the messages $\left\{\mu_{f_{r, m} \rightarrow x_{n}}\left(x_{n}\right)\right\}_{m=n+1}^{n+L}$ from (16) and therefore contribute to the update of messages $\left\{\mu_{x_{n} \rightarrow f_{M, g}}\left(x_{n}\right)\right\}_{n=1}^{N}$ from (20). For example, the Volterra kernels $h_{i j k}, i, \jmath, k \neq 0$ are used for updating the message $\mu_{f_{r, n+1} \rightarrow x_{n}}\left(x_{n}\right)$, as long as neither $i, \jmath$ nor $k$ equals to 1 . By resorting to this approximation, on the order of $\mathcal{O}\left[(L+1)\left(\frac{1}{2} L^{2}(L+1)\right)\right]$ operations of calculating the products of Volterra kernels and the first-order moments of the Sat-DMIM symbols are omitted from the calculations of (20).

Secondly, we propose to approximate the estimates of $\psi_{n, n}^{o \triangleleft \triangleleft, 2}$ and $\psi_{n, n}^{\triangleleft \triangleleft o, 2}$ by

$$
\begin{aligned}
& \hat{\psi}_{n, n}^{o \triangleleft \triangleleft, 2} \approx \underline{\psi}_{n, n}^{o \triangleleft \triangleleft, 2} \triangleq e^{2} \sum_{i=1}^{L} \sum_{\jmath=1}^{L}\left|h_{0 i \jmath}\right|^{2}, \\
& \hat{\psi}_{n, n}^{\triangleleft \triangleleft o, 2} \approx \underline{\psi}_{n, n}^{\triangleleft \triangleleft o, 2} \triangleq e^{2} \sum_{i=1}^{L} \sum_{j \geq i}^{L}\left|h_{i j 0}\right|^{2},
\end{aligned}
$$

where $e$ is the average energy per transmitted Sat-DMIM symbol. In this way, another $\mathcal{O}\left[(L+1)\left(L^{2}+\frac{1}{2} L(L+1)\right)\right]$ operations are further omitted. Note that this is different from the direct approximation of the system model in [27], where $h_{0 i \jmath}, i, \jmath=1, \ldots, L$, and $h_{i \jmath 0}, i, \jmath=1, \ldots, L, \jmath \geq i$, are directly replaced by zeros. As seen in (6), $h_{0 i j}$ and $h_{i j 0}$ still contribute to the update of the parameters $\psi_{n, n}^{o \triangleleft \triangleleft}$ and $\psi_{n, n}^{\triangleleft \triangleleft o}$. Moreover, in our proposed approximation method, the secondorder moments of Sat-DMIM symbols are updated in an iterative fashion, i.e., $E_{b\left(x_{n-i}\right)}\left[\left|x_{n-i}\right|^{2}\right]=\left|\hat{x}_{n-i}\right|^{2}+v_{x_{n-i}}$, $i=1, \ldots, L$. These values are then used for calculating the parameters $\hat{\psi}_{n, n}^{o \triangleleft o, 2}$ and $\hat{\psi}_{n, n}^{o o \triangleleft, 2}$ of the message $\mu_{f_{r, n} \rightarrow x_{n}}\left(x_{n}\right)$. By contrast, the second-order moments of the transmitted symbols - except for the desired one - are approximated by the average energy and are not updated with the aid of iterations in [27]. On the other hand, considering that the a posteriori variances of the variables $\left\{x_{n-i}\right\}_{i=1}^{L}$, i.e., $\left\{v_{x_{n-i}}\right\}_{i=1}^{L}$ are explicitly exploited for updating the message $\mu_{f_{r, n} \rightarrow x_{n}}\left(x_{n}\right)$ through the parameters $\hat{\psi}_{n, n}^{o \triangleleft o, 2}$ and $\hat{\psi}_{n, n}^{o o \triangleleft, 2}$, the approximation in (23) is different from directly approximating the nonlinear terms as Gaussian noise and then using $\left\{v_{x_{n-i}}\right\}_{i=1}^{L}$ merely to compute the equivalent variance of the Gaussian noise process in [35]. It can be found from (22) and (23) that the number of multiplications required for tracking the message $\mu_{x_{n} \rightarrow f_{M, g}}\left(x_{n}\right)$ is reduced from $\mathcal{O}\left(\frac{1}{2} L^{4}+4 L^{3}+\frac{19}{2} L^{2}+6 L\right)$ to $\mathcal{O}\left(\frac{3}{2} L^{3}+7 L^{2}+\frac{11}{2} L\right)$ per Sat-DMIM symbol.

\section{B. Complexity Reduction Using the First-Order Taylor Series Expansion Approximation}

The message $\mu_{x_{n} \rightarrow f_{M, g}}\left(x_{n}\right)$ in (20) represents a nonlinear distribution of variable $x_{n}$ and consists of $\mathcal{L}^{2}$ sufficient statistics of $x_{n}$. By substituting (20) into (10) and (11), we find that the complexity of calculating the belief of the SatDMIM symbol from (9) is $\mathcal{O}\left[\mathcal{L}^{2}\left(P_{A}+P_{B}\right)\right]$. By contrast, the complexity associated with this part of the classical SC signal transmission over linear dispersive channels only grows linearly with the modulation order. To reduce the complexity of this part in Sat-DMIM, we propose to use the firstorder Taylor series expansion for further approximating the messages $\left\{\mu_{f_{r, m} \rightarrow x_{n}}\left(x_{n}\right)\right\}_{m=n}^{n+L}$. Without loss of generality, we only provide the derivation of the approximate message $\mu_{f_{r, n} \rightarrow x_{n}}\left(x_{n}\right)$, noting that the other messages can be updated following similar steps. Firstly, by regarding $x_{n}$ as the desired variable, we rewrite the nonlinear terms associated with $x_{n}$ in the log-likelihood function $\log f_{r, n}\left(x_{n}, \ldots, x_{n-L}, r_{n}\right)$ as

$$
\begin{aligned}
f_{n}\left(x_{n}, x_{n}^{*}\right)=\left|x_{n}\right|^{2} x_{n} h_{000} & +\left|x_{n}\right|^{2}\left(\sum_{i=1}^{L} x_{n-i} h_{0 i 0}\right) \\
+ & x_{n}^{2}\left(\sum_{i=1}^{L} x_{n-i}^{*} h_{00 i}\right) \\
= & x_{n} x_{n} x_{n}^{*} h_{000}+x_{n} x_{n} \psi_{n, n}^{o o \triangleleft}+x_{n} x_{n}^{*} \psi_{n, n}^{o \triangleleft o},
\end{aligned}
$$

which is subject to the constraints $\Re\left\{x_{n}\right\}=\Re\left\{x_{n}^{*}\right\}$ and $\Im\left\{x_{n}\right\}+\Im\left\{x_{n}^{*}\right\}=0$. Here, in contrast to denoting $x_{n} x_{n}^{*}$ by $\left|x_{n}\right|^{2}$ as in (5), we rewrite the sufficient statistics of $x_{n}$ in an expanded form. This seemingly pedantic distinction is necessary for obtaining an analytic expression without encountering meaningless expressions, such as $\partial\left(\left|x_{n}\right|^{2} x_{n}\right) / \partial x_{n}$. To elaborate a little further, upon defining a function $f: \mathbb{C} \rightarrow \mathbb{C}$ with $x^{*}$ as its variable, for example, $f(x)=x^{*}$, it is not analytic, i.e., the derivation of $x^{*}$ with regard to $x$ is not defined in the realms of normal complex-variable theory [47]. Thus the real function $f: \mathbb{C} \rightarrow \mathbb{R}$ given by $f(x)=|x|^{2}$ is not analytic either. Fortunately, the function $f_{n}\left(x_{n}, x_{n}^{*}\right)$ in (24) is analytic with respect to $x_{n}$ and $x_{n}^{*}$ independently in the sense of partial differentiation. However, deriving the complex-variable derivative is quite a challenging task that is subject to the Cauchy-Riemann conditions [48]. To solve this problem, we introduce the following theorem, noting that the detailed proof is provided in [47].

Theorem 1: Let $f: \mathbb{C} \times \mathbb{C} \rightarrow \mathbb{C}$ be a function of both a complex variable $x$ and of its conjugate $x^{*}$, where $x=$ $y+j z, y$ and $z$ are real numbers and $j=\sqrt{-1}$. Then the partial complex-variable derivatives $\partial f / \partial x$ (treating $x^{*}$ as a constant in $f$ ) and $\partial f / \partial x^{*}$ (treating $x$ as a constant in $f$ ) are equivalent to $\frac{1}{2}(\partial f / \partial y-j \partial f / \partial z)$ and $\frac{1}{2}(\partial f / \partial y+j \partial f / \partial z)$, respectively.

Given the above theorem, the cumbersome expressions of complex-variable derivatives derived in the real field can be mathematically represented by compact complex-field expressions for the sake of convenience. Thus, the first-order Taylor 
series expansion of $f_{n}\left(x_{n}, x_{n}^{*}\right)$ at the estimate of $x_{n}$ gives

$$
\delta f_{n}\left(x_{n}, x_{n}^{*}\right) \approx \frac{\partial f_{n}\left(x_{n}, x_{n}^{*}\right)}{\partial x_{n}} \delta x_{n}+\frac{\partial f_{n}\left(x_{n}, x_{n}^{*}\right)}{\partial x_{n}^{*}} \delta x_{n}^{*},
$$

where the partial derivative $\frac{\partial f_{n}\left(x_{n}, x_{n}^{*}\right)}{\partial x_{n}}$ (alternatively, $\frac{\partial f_{n}\left(x_{n}, x_{n}^{*}\right)}{\partial x_{n}^{*}}$ ) is obtained by treating $x_{n}^{*}$ (alternatively, $x_{n}$ ) as a constant in $f_{n}$. By substituting (25) into the function $\log f_{r, n}\left(x_{n}, \ldots, x_{n-L}, r_{n}\right)$ in (15) and using the approximations in (22) and (23), we arrive at the partiallinearization approximation of the message $\mu_{f_{r, n} \rightarrow x_{n}}\left(x_{n}\right)$ in (26), where the parameters $\hat{\xi}_{n, n}, \hat{\zeta}_{n, n}$, and $\hat{\rho}_{n, n}$ are made up of the estimate of $x_{n}$ obtained from the previous iteration, i.e., $\hat{\xi}_{n, n}=2\left|\hat{x}_{n}\right|^{2} h_{000}+2 \hat{x}_{n} \hat{\psi}_{n, n}^{o o \triangleleft}+\hat{x}_{n}^{*} \hat{\psi}_{n, n}^{o \triangleleft o}+h_{0}+\underline{\psi}_{n, n}^{o \triangleleft \triangleleft}$, $\hat{\zeta}_{n, n}=\hat{x}_{n}^{2} h_{000}+\hat{x}_{n} \hat{\psi}_{n, n}^{o \triangleleft o}+\underline{\psi}_{n, n}^{\triangleleft \triangleleft o}$, and $\hat{\rho}_{n, n}=$ $-2\left|\hat{x}_{n}\right|^{2} \hat{x}_{n} h_{000}-\hat{x}_{n}^{2} \hat{\psi}_{n, n}^{o o \triangleleft}-\left|\hat{x}_{n}\right|^{2} \hat{\psi}_{n, n}^{o \triangleleft o}$.

In a similar way, the number of sufficient statistics of $x_{n}$ involved in messages $\left\{\mu_{f_{r, m} \rightarrow x_{n}}\left(x_{n}\right)\right\}_{n+1}^{n+L}$ can also be reduced. After some algebraic manipulations, we find that the preserved sufficient statistics of these messages are the same, which are $\left|x_{n}\right|^{2}, \Re\left\{x_{n}^{2}\right\}$ and $\Re\left\{x_{n}\right\}$. Let us denote the parameters of the message $\mu_{f_{r, m} \rightarrow x_{n}}\left(x_{n}\right)$ corresponding to $\left|x_{n}\right|^{2}, \Re\left\{x_{n}^{2}\right\}$ and $\Re\left\{x_{n}\right\}$ by $\underline{\hat{\varphi}}_{r_{m}, n}^{1,1}, \underline{\hat{\phi}}_{r_{m}, n}^{2,0}$ and $\underline{\phi}_{r_{m}, n}^{1,0}$, respectively. Then the message $\mu_{x_{n} \rightarrow f_{M, g}}\left(x_{n}\right)$ can be approximately updated by ${ }^{4}$

$$
\begin{aligned}
\mu_{x_{n} \rightarrow f_{M, g}}\left(x_{n}\right) \approx \exp & \left\{-\frac{1}{2 \sigma^{2}} \sum_{m=n}^{n+L}\left(\underline{\hat{\varphi}}_{r_{m}, n}^{1,1}\left|x_{n}\right|^{2}\right.\right. \\
+ & \left.\left.2 \Re\left\{\underline{\hat{\phi}}_{r_{m}, n}^{2,0} x_{n}^{2}+\underline{\phi}_{r_{m}, n}^{1,0} x_{n}\right\}\right)\right\} .
\end{aligned}
$$

Using (26) and (27), the complexity order of the decoupling step in Algorithm 1 is reduced to $\mathcal{O}\left[3\left(P_{A}+P_{B}\right)\right]$.

\section{Message Passing Scheduling}

The message passing schedule for the proposed joint SatDMIM symbol detection and decoding algorithm based on the partial-linearization approximation-aided amalgamated BPMF method in nonlinear dispersive satellite channels, which is referred to as PL-BP-MF, is summarized in Algorithm 2.

\begin{tabular}{l}
\hline Algorithm 2 The Proposed Joint Sat-DMIM Symbols De- \\
tection and Decoding Algorithm Based on the Partial- \\
Linearization Approximation-Aided Amalgamated BP-MF \\
Method in Nonlinear Dispersive Channels. \\
\hline 1: The same as BP-MF except for the modifications imposed \\
on the decoupling step: \\
2: - Decoupling step: For $n=1, \ldots, N, m=n, \ldots, n+L$, \\
compute the message $\mu_{f_{r, m} \rightarrow x_{n}}\left(x_{n}\right)$ and $\mu_{x_{n} \rightarrow f_{M, g}}\left(x_{n}\right)$ \\
from (26) and (27), respectively.
\end{tabular}

\section{Complexity Analysis}

In Table II, we briefly compare the complexity of the proposed BP-MF and PL-BP-MF methods to existing equalizers

\footnotetext{
${ }^{4}$ The accuracy of the approximations in (26) and (27) can be improved with iterations.
}

operating in dispersive nonlinear satellite channels. In codeaided systems, all of these methods have to perform standard BCJR decoding [44]. Thus we only focus our attention on the computational complexity of the soft demodulation part. The near-optimal FB equalizer of [28] extended from the conventional Satcom to the proposed Sat-DMIM scheme has a complexity order of $\mathcal{O}\left[\left(P_{A}+P_{B}\right)^{L}\right]$. By ignoring the nonlinear ISI terms, the conventional linear equalizer of [43] requires $\mathcal{O}\left[\beta\left(\beta^{2}+P_{A}+P_{B}\right)\right]$ operations, where $\beta$ is the size of the sliding window. In [36], the BER performance of the linear equalizer is further improved by taking the nonlinear ISI terms into account, at the cost of increasing the complexity order to $\mathcal{O}\left[\beta\left(\beta^{2}+\left(\frac{1}{2} L^{3}+\frac{7}{2} L^{2}+6 L\right)+\left(P_{A}+P_{B}\right)\right)\right]$. For the proposed amalgamated BP-MF method, $\mathcal{O}\left[(L+1)\left(\frac{1}{2} L^{3}+\frac{7}{2} L^{2}+6 L\right)\right]$ operations of calculating the products of the Volterra kernels and the first/second-order moments of Sat-DMIM symbols are required for evaluating (16), and $\mathcal{O}\left(\mathcal{L}^{2}\left(P_{A}+P_{B}\right)\right)$ operations are needed for calculating (10) and (11). With the aid of the partial-linearization approximations in (22), (23) and (25), the total number of multiplications involved in the proposed PL-BP-MF method are reduced to $\mathcal{O}\left(\frac{3}{2} L^{3}+7 L^{2}+\frac{11}{2} L\right)$, and accordingly the complexity order of calculating (10) and (11) is reduced to $\mathcal{O}\left[3\left(P_{A}+P_{B}\right)\right]$. In Table II, we provide an example for $L=3, P_{A}=4, P_{B}=8$ and $\beta=4$. The complexity reduction [49] by adopting the proposed BPMF and PL-BP-MF algorithms compared to the FB is also provided. We observe that the proposed BP-MF and PL-BPMF methods are computationally more efficient than the FB equalizer. The complexity order of BP-MF is slightly lower than that of the existing nonlinear equalizer.

\section{Simulation Results}

In this section, the performance of the proposed iterative detection algorithms for the Sat-DMIM scheme is evaluated by Monte Carlo simulations. In all simulations, a rate- $2 / 3$ (i.e., $R_{c}=2 / 3$ ) LDPC code $^{5}$ having a block size of 6048 is employed. The number of iterations within the LDPC decoder is $T_{\mathrm{LDPC}}=10$, while the maximum number of iterations between the SISO demodulation and SISO decoding is $T_{\operatorname{ex}(\max )}=20$, unless otherwise stated. The tolerance value is set to $\epsilon=10^{-12}$. For characterizing the dispersive nonlinear satellite channels, a third-order Volterra model (termed as "Nonlinear Channel-I") is considered, the coefficients of which were derived in [34] but with stronger nonlinear ISI [36]. The average channel energy is normalized to 1 .

Fig. 3 shows the BERs of the proposed BP-MF algorithm for the Sat-DMIM scheme characterized by $N_{g}=4$, $K_{g}=2, P_{A}=4$ and $P_{B}=8$ (termed as "Sat-DMIM $(4,2,4,8)$ "), i.e., the modes A and B are QPSK and 8PSK constellations, respectively. The Volterra model of Nonlinear Channel-I having a channel memory of $L=3$ symbols is considered [36]. In order to increase the minimum Euclidean distance of the joint constellation set $\dot{\mathcal{S}} \cup \ddot{\mathcal{S}}$, the 8PSK constellation is rotated by a phase of $\pi / 8$ [18]. The

\footnotetext{
${ }^{5}$ The variable and check node degree distributions are $X_{1}=0.00005+$ $0.2043 X+0.5037 X^{2}+0.2902 X^{7}$ and $X_{2}=0.1975 X^{8}+0.8025 X^{9}$ respectively. The parameter definitions follow those of [37].
} 


$$
\begin{aligned}
& \mu_{f_{r, n} \rightarrow x_{n}}\left(x_{n}\right) \propto \exp \left\{E_{b\left(x_{n-1}\right), \ldots, b\left(x_{n-L}\right)}\left[-\frac{1}{2 \sigma^{2}}\left|r_{n}-\left(f_{n}\left(x_{n}, x_{n}^{*}\right)+x_{n}\left(h_{0}+\psi_{n, n}^{o \triangleleft \triangleleft}\right)+x_{n}^{*} \psi_{n, n}^{\triangleleft \triangleleft o}+\varepsilon_{n, n}\right)\right|^{2}\right]\right\} \\
& \approx \exp \left\{E _ { b ( x _ { n - 1 } ) , \ldots , b ( x _ { n - L } ) } \left[-\frac{1}{2 \sigma^{2}} \mid r_{n}-\left(x_{n}\left[2 \hat{x}_{n} \hat{x}_{n}^{*} h_{000}+2 \hat{x}_{n} \psi_{n}^{o o \triangleleft}+\hat{x}_{n}^{*} \psi_{n, n}^{o \triangleleft o}+h_{0}+\psi_{n, n}^{o \triangleleft \triangleleft}\right]\right.\right.\right. \\
& \left.\left.\left.+x_{n}^{*}\left[\hat{x}_{n}^{2} h_{000}+\hat{x}_{n} \psi_{n, n}^{o \triangleleft o}+\psi_{n, n}^{\triangleleft \triangleleft o}\right]-2 \hat{x}_{n}^{2} \hat{x}_{n}^{*} h_{000}-\hat{x}_{n}^{2} \psi_{n, n}^{o o \triangleleft}-\hat{x}_{n} \hat{x}_{n}^{*} \psi_{n, n}^{o \triangleleft o}+\varepsilon_{n, n}\right)\left.\right|^{2}\right]\right\} \\
& \approx \exp \left\{-\frac{1}{2 \sigma^{2}}\left[| x _ { n } | ^ { 2 } \left[5\left|\hat{x}_{n}\right|^{4}\left|h_{000}\right|^{2}+\left|\hat{x}_{n}\right|^{2}\left(2 \hat{\psi}_{n, n}^{o \triangleleft o, 2}+4 \hat{\psi}_{n, n}^{o o \triangleleft, 2}\right)+\left|h_{0}\right|^{2}+\underline{\psi}_{n, n}^{o \triangleleft \triangleleft, 2}+\underline{\psi}_{n, n}^{\triangleleft \triangleleft o, 2}+2 \Re\left\{\left|\hat{x}_{n}\right|^{2} \hat{x}_{n}\left[4 h_{000}^{*} \hat{\psi}_{n, n}^{o o \triangleleft}+3 h_{000} \hat{\psi}_{n, n}^{o \triangleleft o, 2}\right]\right.\right.\right.\right. \\
& \left.\left.+2 \hat{x}_{n}^{2} \hat{\psi}_{n, n}^{o o \triangleleft}\left(\hat{\psi}_{n, n}^{o \triangleleft o}\right)^{*}+2\left|\hat{x}_{n}\right|^{2} h_{000}^{*}\left(h_{0}+\underline{\psi}_{n, n}^{o \triangleleft \triangleleft}\right)+\hat{x}_{n}\left[2 \hat{\psi}_{n, n}^{o o \triangleleft}\left(h_{0}+\underline{\psi}_{n, n}^{o \triangleleft \triangleleft}\right)^{*}+\left(\hat{\psi}_{n, n}^{o \triangleleft o}\right)^{*}\left(h_{0}+\underline{\psi}_{n, n}^{o \triangleleft \triangleleft}\right)+h_{000}\left(\underline{\psi}_{n, n}^{\triangleleft \triangleleft o}\right)^{*}+\hat{\psi}_{n, n}^{o \triangleleft o}\left(\underline{\psi}_{n, n}^{\triangleleft \triangleleft o}\right)^{*}\right]+h_{0}^{*} \underline{\psi}_{n, n}^{o \triangleleft \triangleleft}\right\}\right] \\
& \left.\left.+2 \Re\left\{x_{n}^{2}\left[\hat{\xi}_{n, n} \hat{\zeta}_{n, n}^{*}\right]+x_{n}\left[\hat{\xi}_{n, n}\left(\hat{\rho}_{n, n}+\underline{\hat{\varepsilon}}_{n, n}\right)^{*}+\hat{\zeta}_{n, n}^{*}\left(\hat{\rho}_{n, n}+\underline{\hat{\varepsilon}}_{n, n}\right)-r_{n}^{*} \hat{\xi}_{n, n}-r_{n} \hat{\zeta}_{n, n}^{*}\right]\right\}\right]\right\} \\
& \triangleq \exp \left\{-\frac{1}{2 \sigma^{2}}\left[\underline{\hat{\varphi}}_{r_{n}, n}^{1,1}\left|x_{n}\right|^{2}+2 \Re\left\{\underline{\hat{\phi}}_{r_{n}, n}^{2,0} x_{n}^{2}+\underline{\hat{\phi}}_{r_{n}, n}^{1,0} x_{n}\right\}\right]\right\}
\end{aligned}
$$

TABLE II

COMPARISONS OF COMPUTATIONAL COMPLEXITY (For EXAMPLE: $L=3, P_{A}=4, P_{B}=8$ AND $\beta=4$.) $L$ :Dispersion duration; $P_{A}$ : Modulation order of Mode A; $P_{B}$ :Modulation order of Mode $\mathrm{B} ; \beta$ :Size OF SLiding Window

\begin{tabular}{|l|r|r|r|}
\hline Algorithm & Complexity of the demodulator & Value & Complexity reduction compared to FB [28] \\
\hline BP-MF & $\mathcal{O}\left((L+1)\left(\frac{1}{2} L^{3}+\frac{7}{2} L^{2}+6 L\right)+\mathcal{L}^{2}\left(P_{A}+P_{B}\right)\right)$ & $\mathcal{O}(360)$ & $78.8 \%$ \\
\hline PL-BP-MF & $\mathcal{O}\left(\frac{3}{2} L^{3}+7 L^{2}+\frac{11}{2} L+3\left(P_{A}+P_{B}\right)\right)$ & $\mathcal{O}(156)$ & $90.8 \%$ \\
\hline FB [28] & $\mathcal{O}\left(\left(P_{A}+P_{B}\right)^{L}\right)$ & $\mathcal{O}\left(1.7 \times 10^{3}\right)$ & - \\
\hline Linear MMSE [43] & $\mathcal{O}\left(\beta\left(\beta^{2}+P_{A}+P_{B}\right)\right)$ & $\mathcal{O}(112)$ & $93.3 \%$ \\
\hline Nonlinear MMSE [36] & $\mathcal{O}\left(\beta\left(\beta^{2}+\left(\frac{1}{2} L^{3}+\frac{7}{2} L^{2}+6 L\right)+\left(P_{A}+P_{B}\right)\right)\right)$ & $\mathcal{O}(364)$ & $78.6 \%$ \\
\hline
\end{tabular}

TABLE III

Comparisons of COMPUTATIONAL COMPLEXITY OF DiFFERENT SCHEMES (For EXAMPLE: $L=3, P_{A}=4, P_{B}=8$ AND $\beta=4$.) $L$ :Dispersion duration; $P_{A}$ : Modulation order of Mode A; $P_{B}$ :Modulation order of Mode $\mathrm{B} ; \beta$ :Size of SLiding window

\begin{tabular}{|l|r|r|r|r|}
\hline Schemes & Complexity of the demodulator in BP-MF & Value & Complexity of the demodulator in PL-BP-MF & Value \\
\hline Sat-DMIM & $\mathcal{O}\left[(L+1)\left(\frac{1}{2} L^{3}+\frac{7}{2} L^{2}+6 L\right)+\mathcal{L}^{2}\left(P_{A}+P_{B}\right)\right]$ & $\mathcal{O}(360)$ & $\mathcal{O}\left(\frac{3}{2} L^{3}+7 L^{2}+\frac{11}{2} L+3\left(P_{A}+P_{B}\right)\right)$ & $\mathcal{O}(156)$ \\
\hline Classical Satcom & $\mathcal{O}\left[(L+1)\left(\frac{1}{2} L^{3}+\frac{7}{2} L^{2}+6 L\right)+\mathcal{L}^{2} P_{\mathcal{S}}\right]$ & $\mathcal{O}(324)$ & $\mathcal{O}\left[\left(\frac{3}{2} L^{3}+7 L^{2}+\frac{11}{2} L\right)+3 P_{\mathcal{S}}\right]$ & $\mathcal{O}(144)$ \\
\hline Sat-IM & $\mathcal{O}\left[(L+1)\left(\frac{1}{2} L^{3}+\frac{7}{2} L^{2}+6 L\right)+\mathcal{L}^{2}\left(P_{\mathrm{IM}}+1\right)\right]$ & $\mathcal{O}(549)$ & $\mathcal{O}\left[\left(\frac{3}{2} L^{3}+7 L^{2}+\frac{11}{2} L\right)+3\left(P_{\mathrm{IM}}+1\right)\right]$ & $\mathcal{O}(219)$ \\
\hline
\end{tabular}

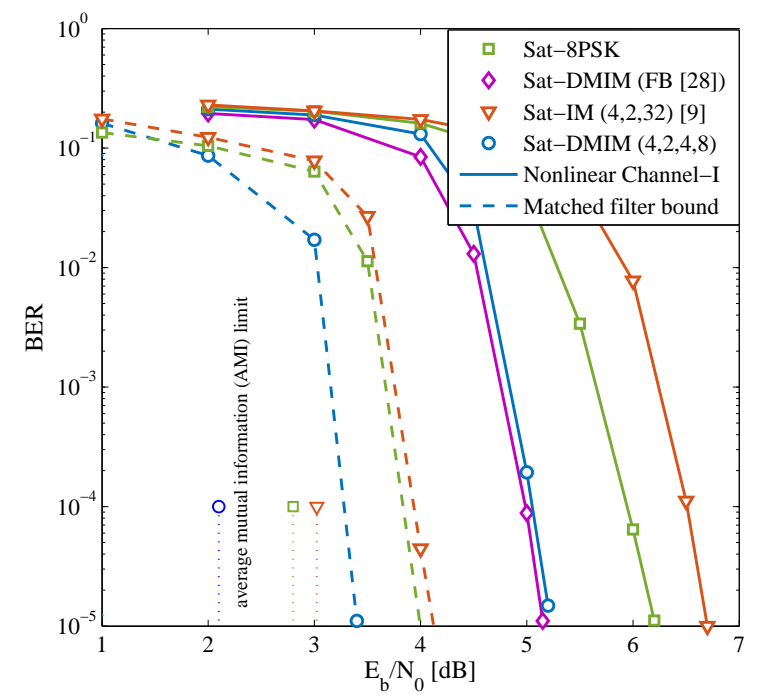

Fig. 3. BER performance of classical 8 PSK modulated Satcom, Sat-DMIM (characterized by "( $4,2,4,8)$ ") and Sat-IM (characterized by " $(4,2,32)$ ") systems communicating over nonlinear dispersive satellite channels modeled by Volterra model "Nonlinear Channel-I" [36]. Both the proposed BP-MF and the existing FB [28] methods are evaluated. The effective throughput is 2 bits/symbol. The number of iterations is fixed at $\left\{T_{\mathrm{LDPC}}=10, T_{\mathrm{ex}(\max )}=\right.$ $20\}$.

constellation design of the Sat-DMIM $(4,2,4,8)$ scheme is $\dot{\mathcal{S}}=\{0.5671+j 0,0+j 0.5671,-0.5671+j 0,0-j 0.5671\}$ and $\overrightarrow{\mathcal{S}}=\{1.1974+j 0.4960,0.4960+j 1.1974,-0.4960+$ $j 1.1974,-1.1974+j 0.4960,-1.1974-j 0.4960,-0.4960-$ $j 1.1974,0.4960-j 1.1974,1.1974-j 0.4960\}$, where the average transmit power of the joint constellation is fixed to unity. For comparison, the BER curve of conventional 8PSK modulated Satcom (denoted by "Sat-8PSK"), yielding the same transmission rate as that of Sat-DMIM $(4,2,4,8)$, is plotted. Additionally, the BER of Satcom relying on the single-mode-aided IM [9] (Sat-IM) is also provided. In the Sat-IM system, only 2 out of 4 available indices are activated for transmitting 32QAM symbols (denoted by "Sat-IM $(4,2,32)$ "). The complexity comparison of the aforementioned three schemes under the conditions of Fig. 3 is given in Table III. The matched filter bounds of the aforementioned three schemes are also shown, serving as benchmarks. Considering that the FB equalizer of [28] is deemed to strike one of the best performance vs. complexity trade-off in the Satcom literature, the BER curve of Sat-DMIM relying on the FB equalizer (denoted by "Sat-DMIM (FB)") is also shown for comparison. As seen from Fig. 3, in terms of matched filter bound, the DM-aided IM scheme outperforms its single-mode-aided and conventional single-carrier counterparts in the high-SNR region, in agreement with the findings of [17], [18], [21]. This confirms the BER advantage of the bits conveyed in the DMaided IM domain [6]. In dispersive nonlinear satellite channels, the proposed BP-MF algorithm approaches the FB equalizer, despite its much lower computational complexity (see Table. II), which confirms the efficiency of our amalgamated message passing method based iterative detection algorithm. Observe 


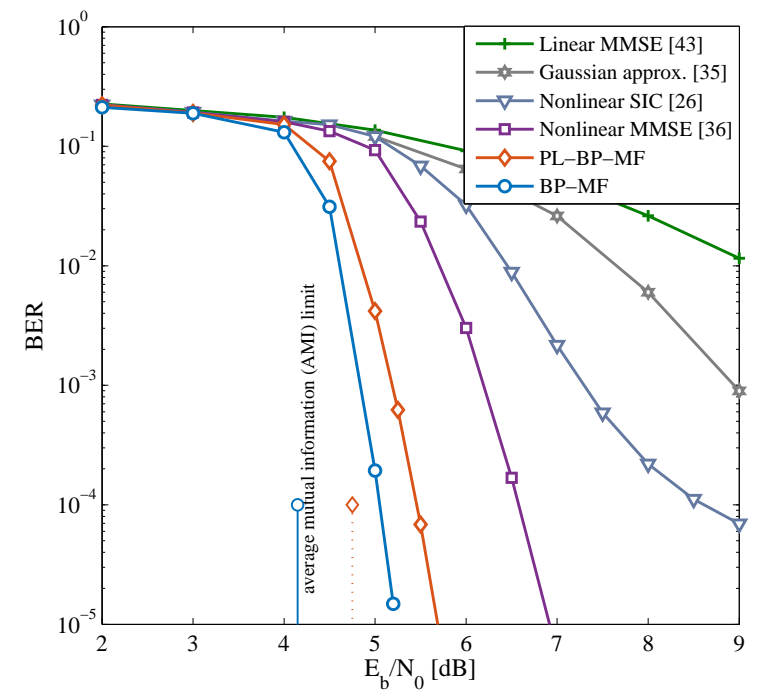

Fig. 4. The BER performance of the proposed BP-MF and PL-BP-MF compared with existing Linear MMSE [43], Nonlinear MMSE [36], Nonlinear SIC [26] and Gaussian approx. [35] algorithms. The Sat-DMIM system (characterized by " $(4,2,4,8)$ ") communicates over nonlinear dispersive satellite channels modeled by Volterra model "Nonlinear Channel-I" [36]. The effective throughput is $2 \mathrm{bits} / \mathrm{symbol}$. The number of iterations is fixed at $\left\{T_{\mathrm{LDPC}}=10, T_{\mathrm{ex}(\max )}=20\right\}$.

furthermore in Fig. 3 that similar to the phenomenon shown in [18] for single-carrier systems over linear dispersive channels, the BER degradation of Sat-DMIM with regard to the freeISI bound is lower than that of Sat-IM and Sat-8PSK for transmission over dispersive nonlinear channels. More specifically, the performance advantage of the Sat-DMIM $(4,2,4,8)$ scheme over the conventional Sat-8PSK and Sat-IM $(4,2,32)$ is approximately $1 \mathrm{~dB}$ and $1.5 \mathrm{~dB}$, respectively, if the target BER is $10^{-5}$. This means that using the proposed BP-MF algorithm, the Sat-DMIM scheme's capability of transmitting a pair of distinguishable constellation modes indeed succeeds in improving the performance over conventional Satcom at a slightly higher complexity (see Table III), despite encountering dispersive channels and nonlinear distortions. Note that although the Sat-IM scheme also implicitly conveys additional information using the active indices in the IM domain, there exist some deactivated time slots within each subframe [9], which significantly limits the spectral efficiency of Sat-IM. Therefore, Sat-IM has to employ the highest-order modulation amongst the aforementioned schemes and thus exhibits the worst BER performance, since higher order modulations tend to experience more grave nonlinear distortions over satellite channels [1].

In Fig. 4, the BER performance of the proposed BP-MF and PL-BP-MF based iterative detection algorithms is compared to that of the linear MMSE equalizer of [43] (denoted by "Linear MMSE"), nonlinear equalizer of [36] (denoted by "Nonlinear MMSE") and nonlinear soft interference canceler (SIC) of [26] (denoted by "Nonlinear SIC"). Additionally, the BER curve of the Gaussian noise approximation based approach of [35] (denoted by "Gaussian approx.") is also shown for comparison. The system parameters are the same as in Fig. 3. It is seen that the Sat-DMIM performance degrades significantly, if the effect of nonlinear distortions is ignored.
By regarding the nonlinear channels as additive Gaussian noise channel associated with a signal attenuation, the approach of [35] delivers better BER performance compared to the linear MMSE receiver. However, the performance loss imposed by this method is still considerable, because the Gaussian noise channel approximation substantially deviates from the actual channel. The nonlinear SIC of [26] improves the Sat-DMIM performance by explicitly taking part of the nonlinear ISI terms into account. We point out that the SIC method may be viewed as a special case of the nonlinear MMSE equalizer of [36], where the third-order interference terms are ignored when computing the variance of the residual equalizer output error. This explains the poor BER performance of nonlinear SIC compared to nonlinear MMSE at high SNRs, where the performance is mainly limited by the nonlinear distortions instead of the additive white noise. Explicitly, it suffers from an error floor at $\mathrm{BER}=10^{-4}$. By using the nonlinear MMSE method, the BER performance improves considerably at the expense of an increased of complexity (see Table II). However, it is inferior to the proposed BP-MF and PL-BP-MF methods. One of the reasons is that the nonlinear MMSE only utilizes the first- and second-order moments of data symbols, ignoring the structured a priori information of the Sat-DMIM signal, which is inherent in the DM-aided IM scheme. By contrast, the proposed BP-MF and PL-BP-MF methods take full account of the mode-selection pattern constraint and explicitly construct the structured a priori probabilities of the Sat-DMIM signal using the extrinsic information provided by the SISO decoder from the previous iteration, see (7) and (8). The other reason is that the expectation of the product of two symbol vectors having different indices is approximated to be zero in the nonlinear MMSE equalizer. In other words, the expectations of the parameters $\psi_{n, n}^{o \triangleleft \triangleleft}$ and $\psi_{n, n}^{\triangleleft \triangleleft o}$ are always set to zero and they are not updated throughout the iterations. By contrast, in the proposed BP-MF and PL-BP-MF algorithms, these two parameters are iteratively updated for calculating the messages passed from the variable node $x_{n}$ to the observation node $f_{r_{m}}$. We also observe that compared to BP-MF (without ignoring high-order terms), the BER performance degradation of the proposed low-complexity PL-BP-MF (with ignoring some high-order terms) is modest, which confirms the efficiency of the first-order Taylor series expansion approximation of the nonlinear message $\mu_{f_{r, n} \rightarrow x_{n}}\left(x_{n}\right)$ following from (26) and the simplification of the message parameters according to (22) and (23).

Furthermore, Fig. 5 plots the BERs of the iterative detection algorithms as a function of iteration index $T_{\mathrm{ex}}$ (the number of iterations between the SISO demodulation and the LDPC decoding) under the conditions of Fig. 4. The SNR is fixed to $5.2 \mathrm{~dB}$. Observe that there is an evident performance improvement, upon increasing the number of iterations. As shown in Fig. 5, the proposed BP-MF algorithm converges after about 5 iterations. This outcome indicates that 5 iterations on average are sufficient to meet the pre-defined stopping criterion. The convergence rate of the proposed low-complexity PL-BP-MF is slighter lower. This outcome may be attributed to the fact that the approximations of the message parameters in the PLBP-MF algorithm introduce some performance loss compared 


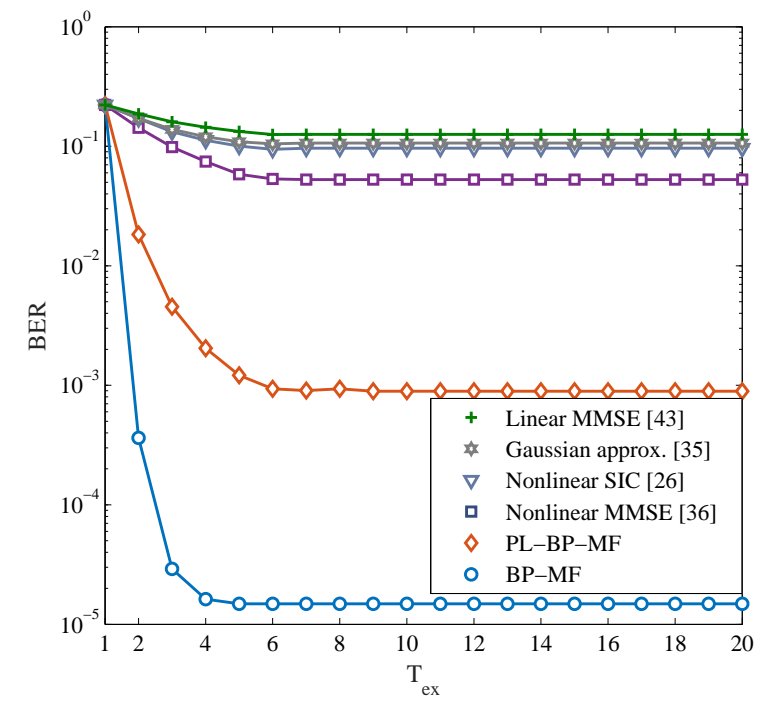

Fig. 5. The BER performance of the proposed BP-MF and PL-BPMF algorithms and existing methods versus iteration index $T_{\text {ex }}$. The SatDMIM system (characterized by " $(4,2,4,8)$ ") communicates over nonlinear dispersive satellite channels modeled by Volterra model "Nonlinear ChannelI" [36]. The effective throughput is 2 bits/symbol. The number of iterations is fixed at $T_{\mathrm{LDPC}}=10$.

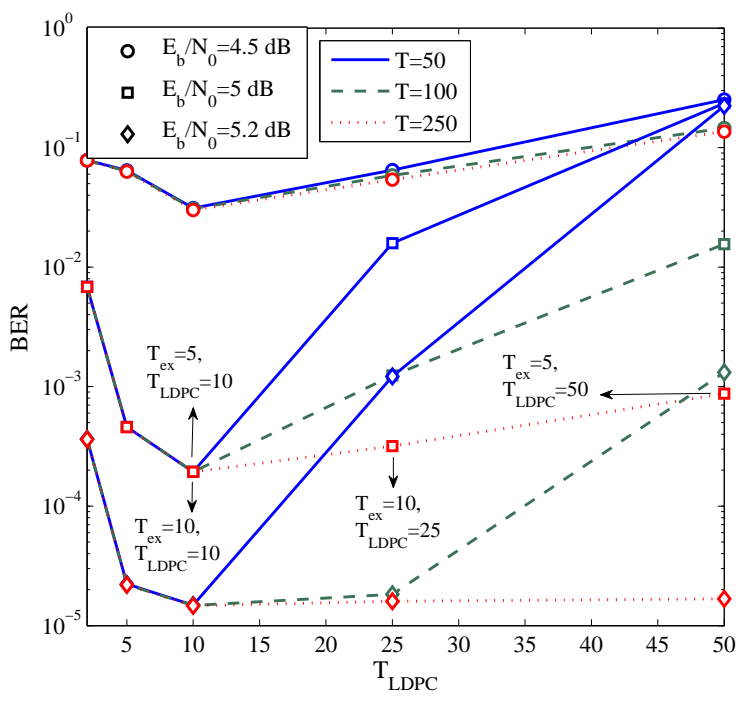

Fig. 6. The BER performance of the proposed BP-MF algorithm versus the number of iterations within the LDPC decoder. The Sat-DMIM system (characterized by " $(4,2,4,8)$ ") communicates over nonlinear dispersive satellite channels modeled by Volterra model "Nonlinear Channel-I" [36]. The effective throughput is 2 bits/symbol.

to BP-MF. Additionally, the convergence of the nonlinear MMSE equalizer is slow compared to the proposed BP-MF, which explains its poor BER performance at high SNRs as seen in Fig. 4.

In Fig. 6, we evaluate the BER performance of the proposed BP-MF algorithm with various combinations of $T_{\mathrm{LDPC}}$ and $T_{\text {ex }}$, where the total number of iterations is $T=T_{\mathrm{LDPC}} \cdot T_{\mathrm{ex}}$. The SNRs $4.5 \mathrm{~dB}, 5 \mathrm{~dB}$ and $5.2 \mathrm{~dB}$ are considered. As shown in Fig. 6, when $T_{\text {ex }} \leq 5$, an improved BER is attained by increasing $T_{\mathrm{ex}}$ at a fixed $T_{\mathrm{LDPC}}$, see $\left\{T_{\mathrm{LDPC}}=50, T_{\mathrm{ex}}=1\right\}$ and $\left\{T_{\mathrm{LDPC}}=50, T_{\mathrm{ex}}=2\right\}$ at $E_{b} / N_{0}=5.2 \mathrm{~dB}$. However, there will be no further performance gain from subsequent $T_{\mathrm{ex}}$

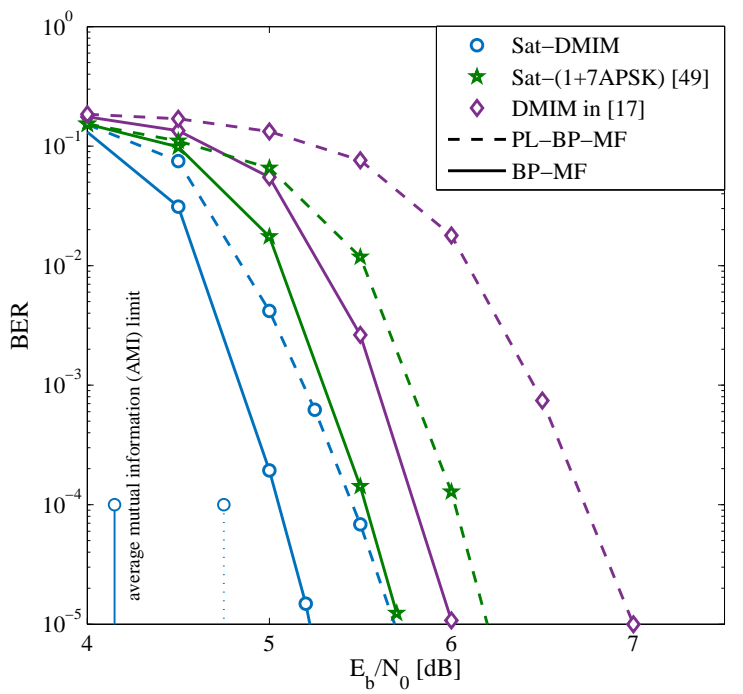

Fig. 7. BER performance of Sat-DMIM (characterized by “( $4,2,4,8)$ )"), $1+7$ APSK modulated Satcom [50] and Sat-DMIM using the mode-selection mapping method of [17] communicating over nonlinear dispersive satellite channels modeled by Volterra model "Nonlinear Channel-I" [36]. Both proposed BP-MF and PL-BP-MF methods are studied for comparison. The number of iterations is fixed at $\left\{T_{\mathrm{LDPC}}=10, T_{\mathrm{ex}(\max )}=20\right\}$.

iterations, see $\left\{T_{\mathrm{LDPC}}=10, T_{\mathrm{ex}}=5\right\},\left\{T_{\mathrm{LDPC}}=10, T_{\mathrm{ex}}=\right.$ $10\}$ and $\left\{T_{\mathrm{LDPC}}=10, T_{\mathrm{ex}}=25\right\}$. This trend is similar to that in Fig. 5. By contrast, when $T_{\mathrm{LDPC}} \geq 10$, some performance loss may be introduced if $T_{\mathrm{LDPC}}$ is too large at a fixed $T_{\mathrm{ex}}$, see $\left\{T_{\mathrm{LDPC}}=10, T_{\mathrm{ex}}=5\right\}$ and $\left\{T_{\mathrm{LDPC}}=50, T_{\mathrm{ex}}=5\right\}$ at $E_{b} / N_{0}=5 \mathrm{~dB}$. We observe that $T_{\mathrm{LDPC}}=10$ is sufficient for the proposed BP-MF method. This can be explained by the fact that the LDPC decoder tends to be over-confident when $T_{\text {LDPC }}$ is large. Finally, using an unnecessarily high number of LDPC iterations slows down its operation and dissipates more power.

In Fig. 7, we apply our reduced-complexity PL-BP-MF iterative detection algorithm to the Sat-DMIM $(4,2,4,8)$ scheme and compare the BER performance attained to that of the conventional Satcom relying on an optimized $1+7$ APSK constellation (denoted by "Sat-(1+7APSK)") that was proposed in [50]. Additionally, the BER curve of the existing DM-aided IM scheme applying the mode-selection mapping method of [17] (denoted by "DMIM in [17]") is also shown in Fig. 7 for comparison. We can observe from Fig. 7 that the there is an evident performance improvement when applying the DMIM technique using an optimized mode-selection mapping method in nonlinear channels. Specifically, the Sat-DMIM scheme achieves about $0.5 \mathrm{~dB}$ better performance than the Satcom system using $1+7$ APSK at a BER of $10^{-5}$. The single-carrier system using the DMIM scheme of [17] has the worst BER performance amongst the aforementioned schemes, due to the error propagation introduced by the mapping rule applied between the IM bits and mode-selection patterns. More specifically, an incorrect detection of IM bits usually results in an incorrect detection of the PSK/QAM symbols. As a result, the BER performance of the DMIM scheme of [17] is degraded, especially for different constellation orders in the presence of nonlinear distortions.

Fig. 8 shows the extrinsic information transfer chart (EX- 


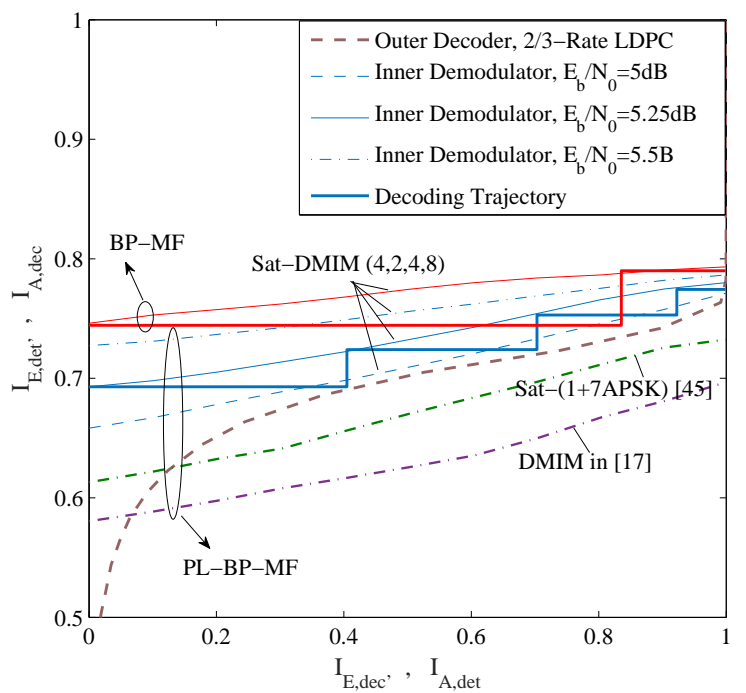

Fig. 8. The EXIT charts of Sat-DMIM (characterized by “( $4,2,4,8)$ ”), $1+$ 7APSK modulated Satcom [50] and Sat-DMIM relying on the proposed BPMF and PL-BP-MF methods. The decoding trajectories are portrayed between the inner demodulator's EXIT curves and the outer LDPC decoder's EXIT curve at $E_{b} / N_{0}=5.25 \mathrm{~dB}$. The number of iterations is fixed at $T_{\mathrm{LDPC}}=$ 10 .

IT $)^{6}$ curves [51] of the Sat-DMIM $(4,2,4,8)$ scheme's inner demodulator using the proposed PL-BP-MF method for SNR values spanning from $5 \mathrm{~dB}$ to $=5.5 \mathrm{~dB}$ with a step size of 0.25 dB. The EXIT curves of the existing Sat-(1 + 7APSK) [50] and DMIM schemes relying on the mode-selection mapping method of [17] used in Fig. 7 are plotted at $\mathrm{SNR}=5.5$ $\mathrm{dB}$ for comparison. The EXIT curve of the $2 / 3$-rate LDPC decoder is also portrayed. Additionally, Fig. 8 shows the Monte-Carlo simulation-based decoding trajectories of the SatDMIM $(4,2,4,8)$ system visualizing the extrinsic information exchange between the inner demodulator and the outer LDPC decoder recorded at $\mathrm{SNR}=5.25 \mathrm{~dB}$ for the proposed $\mathrm{BP}$ MF and PL-BP-MF, respectively. Observe that using the PLBP-MF method advocated, there is no open tunnel between the inner decoder's curve and the outer decoder's curve at $\mathrm{SNR}=5.5 \mathrm{~dB}$ for the Sat-(1 + 7APSK) and DMIM scheme of [17]. By contrast, an open tunnel is seen in the Sat-DMIM $(4,2,4,8)$ system for SNRs beyond $5 \mathrm{~dB}$. According to the open tunnel recorded at $\mathrm{SNR}=5.25 \mathrm{~dB}$, the $\mathrm{BP}-\mathrm{MF}$ iterative detector is expected to converge faster than PL-BP-MF.

\section{CONCLUSIONS}

We proposed an amalgamated BP-MF message passing algorithm based iterative detector for single-carrier systems relying on DM-aided IM communicating over dispersive nonlinear satellite channels. By taking full account of the mode-selection pattern constraint, the structured nature of the a priori information of the Sat-DMIM signal gleaned from the SISO decoder was explicitly exploited. Based on this, the

\footnotetext{
${ }^{6}$ The EXIT charts allow us to analyze the convergence behavior of iteratively decoded systems, where the exchange of extrinsic information between the inner demodulator and the LDPC decoder can be visualized by calculating the mutual information between hard-decision based information bits and the soft values [51]. The $E_{b} / N_{0}$ at which a semi-convergent tunnel starts to appear is referred to as the average mutual information (AMI) limit.
}

associated factor graph was conceived by factorizing the joint a posteriori pdf of the information bits, of the coded bits and of the Sat-DMIM symbols. The BP method was applied both at the mode-selection pattern constraint nodes and at the conventional PSK/QAM modulation constraint nodes, while the MF technique was invoked at the observation nodes for decoupling the Sat-DMIM symbols. For further reducing the complexity, the complicated nonlinear messages are updated by simplifying the message parameters and approximating the high-order ISI terms using the first-order Taylor series expansion method. Our simulation results showed that the proposed BP-MF approaches the FB equalizer's performance, despite its lower computational complexity, as shown in Table II. Compared to the existing linear MMSE, nonlinear MMSE, nonlinear SIC and Gaussian noise approximation based methods which were originally designed for conventional Satcom and then were extended here to the Sat-DMIM schemes, the proposed methods beneficially improve the BER performance. We also demonstrated the robustness of the proposed SatDMIM scheme compared to its conventional Satcom and single-mode-aided Sat-IM counterparts.

\section{REFERENCES}

[1] M. Jia, X. Gu, Q. Guo, W. Xiang, and N. Zhang, "Broadband hybrid satellite-terrestrial communication systems based on cognitive radio toward 5G," IEEE Wireless Commun., vol. 23, no. 6, pp. 96-106, December 2016.

[2] J. P. Choi and C. Joo, "Challenges for efficient and seamless spaceterrestrial heterogeneous networks," IEEE Commun. Mag., vol. 53, no. 5, pp. 156-162, May 2015.

[3] L. Kuang, X. Chen, C. Jiang, H. Zhang, and S. Wu, "Radio resource management in future terrestrial-satellite communication networks," IEEE Wireless Commun., vol. 24, no. 5, pp. 81-87, October 2017.

[4] Z. Lin, M. Lin, J. Wang, T. de Cola, and J. Wang, "Joint beamforming and power allocation for satellite-terrestrial integrated networks with non-orthogonal multiple access," IEEE J. Sel. Topics Signal Process, vol. 13 , no. 3, pp. 657-670, June 2019.

[5] X. Zhu, C. Jiang, L. Kuang, N. Ge, and J. Lu, "Non-orthogonal multiple access based integrated terrestrial-satellite networks," IEEE J. Sel. Areas Commun, vol. 35, no. 10, pp. 2253-2267, Oct 2017.

[6] E. Basar, "Index modulation techniques for $5 \mathrm{G}$ wireless networks," IEEE Commun. Mag., vol. 54, no. 7, pp. 168-175, July 2016.

[7] P. Yang, M. Di Renzo, Y. Xiao, S. Li, and L. Hanzo, "Design guidelines for spatial modulation," IEEE Commun. Surveys Tuts., vol. 17, no. 1, pp. 6-26, Firstquarter 2015.

[8] E. Basar, U. Aygolu, E. Panayirci, and H. V. Poor, "Orthogonal frequency division multiplexing with index modulation," IEEE Trans. Signal Process., vol. 61, no. 22, pp. 5536-5549, Nov 2013.

[9] M. Nakao, T. Ishihara, and S. Sugiura, "Single-carrier frequency-domain equalization with index modulation," IEEE Commun. Lett., vol. 21, no. 2, pp. 298-301, Feb 2017.

[10] J. Choi, "Single-carrier index modulation for IoT uplink," IEEE J. Sel. Topics Signal Process., vol. 13, no. 6, pp. 1237-1248, 2019.

[11] J. B. Anderson, F. Rusek, and V. wall, "Faster-than-Nyquist signaling," Proc. IEEE, vol. 101, no. 8, pp. 1817-1830, Aug 2013.

[12] E. Basar, M. Wen, R. Mesleh, M. Di Renzo, Y. Xiao, and H. Haas, "Index modulation techniques for next-generation wireless networks," IEEE Access, vol. 5, pp. 16693-16746, 2017.

[13] N. Ishikawa, S. Sugiura, and L. Hanzo, "Subcarrier-index modulation aided OFDM - will it work?" IEEE Access, vol. 4, pp. 2580-2593, 2016.

[14] H. Zhang, L. Yang, and L. Hanzo, "Compressed sensing improves the performance of subcarrier index-modulation-assisted OFDM," IEEE Access, vol. 4, pp. 7859-7873, 2016.

[15] T. Ishihara and S. Sugiura, "Faster-than-Nyquist signaling with index modulation,” IEEE Wireless Commun. Lett., vol. 6, no. 5, pp. 630-633, Oct 2017.

[16] C. R. Kumar and R. K. Jeyachitra, "Dual-mode generalized spatial modulation MIMO for visible light communications," IEEE Commun. Lett., vol. 22, no. 2, pp. 280-283, Feb 2018. 
[17] T. Mao, Z. Wang, Q. Wang, S. Chen, and L. Hanzo, "Dual-mode index modulation aided OFDM," IEEE Access, vol. 5, pp. 50-60, 2017.

[18] M. Nakao, T. Ishihara, and S. Sugiura, "Dual-mode time-domain index modulation for Nyquist-criterion and faster-than-Nyquist single-carrier transmissions," IEEE Access, vol. 5, pp. 27 659-27667, 2017.

[19] M. Wen, E. Basar, Q. Li, B. Zheng, and M. Zhang, "Multiple-mode orthogonal frequency division multiplexing with index modulation," IEEE Trans. Commun., vol. 65, no. 9, pp. 3892-3906, Sep. 2017.

[20] M. Wen, Q. Li, E. Basar, and W. Zhang, "Generalized multiple-mode OFDM with index modulation," IEEE Trans. on Wireless Commun., vol. 17 , no. 10 , pp. $6531-6543,2018$.

[21] X. Li, H. Wang, N. Guan, and W. Lai, "A dual-mode index modulation scheme with Gray-coded pairwise index mapping," IEEE Commun. Lett., vol. 22, no. 8, pp. 1580-1583, 2018.

[22] B. Shamasundar, S. Bhat, S. Jacob, and A. Chockalingam, "Multidimensional index modulation in wireless communications," IEEE Access, vol. 6, pp. 589-604, 2018.

[23] P. Yang, Y. Xiao, Y. L. Guan, K. Hari, A. Chockalingam, S. Sugiura, H. Haas, M. Di Renzo, C. Masouros, Z. Liu, L. Xiao, S. Li, and L. Hanzo, "Single-carrier SM-MIMO: A promising design for broadband large-scale antenna systems," IEEE Commun. Surveys Tuts., vol. 18, no. 3, pp. 1687-1716, 2016.

[24] M. D. Renzo, H. Haas, and P. M. Grant, "Spatial modulation for multiple-antenna wireless systems: a survey," IEEE Commun. Mag., vol. 49, no. 12, pp. 182-191, 2011

[25] A. Gutierrez and W. E. Ryan, "Performance of volterra and MLSD receivers for nonlinear band-limited satellite systems," IEEE Trans. Commun., vol. 48, no. 7, pp. 1171-1177, July 2000

[26] D. Ampeliotis, A. A. Rontogiannis, K. Berberidis, M. Papaleo, and G. E. Corazza, "Turbo equalization of non-linear satellite channels using soft interference cancellation," in 2008 4th Advanced Satellite Mobile Systems Conf., Aug 2008, pp. 289-292.

[27] G. Colavolpe and A. Piemontese, "Novel SISO detection algorithms for nonlinear satellite channels," IEEE Wireless Commun. Lett, , vol. 1, no. 1 , pp. 22-25, February 2012.

[28] F. M. Kashif, H. Wymeersch, and M. Z. Win, "Monte Carlo equalization for nonlinear dispersive satellite channels," IEEE J. Sel. Areas Commun, vol. 26, no. 2, pp. 245-255, February 2008.

[29] Z. Long, N. Wu, H. Wang, and Q. Guo, "Turbo equalization based on a combined VMP-BP algorithm for nonlinear satellite channels," IEEE Access, vol. 6, pp. 35 492-35 500, 2018.

[30] X. Wen, W. Yuan, D. Yang, N. Wu, and J. Kuang, "Low complexity message passing receiver for faster-than-nyquist signaling in nonlinear channels," IEEE Access, vol. 6, pp. 68 233-68 241, 2018.

[31] B. Li, C. Zhao, M. Sun, H. Zhang, Z. Zhou, and A. Nallanathan, "A Bayesian approach for nonlinear equalization and signal detection in millimeter-wave communications," IEEE Trans. Wireless Commun., vol. 14, no. 7, pp. 3794-3809, 2015.

[32] F. R. Kschischang, B. J. Frey, and H. A. Loeliger, "Factor graphs and the sum-product algorithm," IEEE Trans. Inform. Theory, vol. 47, no. 2, pp. 498-519, Feb. 2001.

[33] A. Doucet and XD Wang, "Monte Carlo methods for signal processing: a review in the statistical signal processing context," IEEE Signal Process. Mag., vol. 22, no. 6, pp. 152-170, Nov 2005.

[34] S. Benedetto, E. Biglieri, and R. Daffara, "Modeling and performance evaluation of nonlinear satellite Links-A Volterra series approach," IEEE Trans. Aerosp. Electron. Syst., vol. AES-15, no. 4, pp. 494-507, July 1979.

[35] C. E. Burnet and W. G. Cowley, "Performance analysis of turbo equalization for nonlinear channels," in Proceedings. International Symposium on Information Theory, 2005. ISIT 2005., Sep. 2005, pp. 2026-2030.

[36] B. Benammar, N. Thomas, C. Poulliat, M. Boucheret, and M. Dervin, "On linear MMSE based turbo-equalization of nonlinear Volterra channels," in Proc. Int. Conf. Acoust., Speech, Signal Process. (ICASSP), May 2013, pp. 4703-4707.

[37] J. Xu, L. Chen, I. Djurdjevic, S. Lin, and K. Abdel-Ghaffar, "Construction of regular and irregular LDPC codes: Geometry decomposition and masking," IEEE Trans. Inf. Theory, vol. 53, no. 1, pp. 121-134, Jan 2007.

[38] E. ETSI, "Digital video broadcasting (dvb); second generation framing structure, channel coding and modulation systems for broadcasting, interactive services, news gathering and other broadband satellite applications," Technical report, Tech. rep., ETSI, Tech. Rep., 2005.

[39] Lei Ding, G. T. Zhou, D. R. Morgan, Zhengxiang Ma, J. S. Kenney, Jaehyeong Kim, and C. R. Giardina, "A robust digital baseband predistorter constructed using memory polynomials," IEEE Trans. on Commun., vol. 52, no. 1, pp. 159-165, 2004.
[40] Er-Wei Bai and Minyue Fu, "A blind approach to Hammerstein model identification," IEEE Trans. Signal Process., vol. 50, no. 7, pp. 16101619, 2002.

[41] G. Colavolpe, A. Modenini, and F. Rusek, "Channel shortening for nonlinear satellite channels," IEEE Commun. Lett., vol. 16, no. 12, pp. 1929-1932, 2012.

[42] J. Zhang, C. Guo, J. Liu, X. Wu, A. P. T. Lau, C. Lu, and S. Yu, "Decision-feedback frequency-domain Volterra nonlinear equalizer for IM/DD OFDM long-reach PON," J. Lightw. Technol., vol. 37, no. 13, pp. 3333-3342, 2019.

[43] M. Tuchler, R. Koetter, and A. C. Singer, "Turbo equalization: principles and new results," IEEE Trans. Commun., vol. 50, no. 5, pp. 754-767, May 2002.

[44] V. Franz and J. B. Anderson, "Concatenated decoding with a reducedsearch BCJR algorithm," IEEE J. Sel. Areas Commun., vol. 16, no. 2, pp. 186-195, Feb 1998.

[45] E. Riegler, G. E. Kirkelund, C. N. Manchon, M. Badiu, and B. H. Fleury, "Merging belief propagation and the mean field approximation: A free energy approach," IEEE Trans. Inf. Theory, vol. 59, no. 1, pp. 588-602, Jan. 2013.

[46] B. F. Beidas, "Intermodulation distortion in multicarrier satellite systems: Analysis and turbo Volterra equalization," IEEE Trans. on Commun., vol. 59, no. 6, pp. 1580-1590, 2011.

[47] D. H. Brandwood, "A complex gradient operator and its application in adaptive array theory," IEE Proc. F, Commun., Radar Signal Process., vol. 130, no. 1, pp. 11-16, 1983.

[48] W. Wirtinger, "Zur formalen Theorie der Funktionen von mehr komplexen Veränderlichen," Mathematische Annalen, vol. 97, no. 1, pp. 357-375, 1927.

[49] J. Li, Q. Li, S. Dang, M. Wen, X.-Q. Jiang, and Y. Peng, "Lowcomplexity detection for index modulation multiple access," IEEE Wireless Commun. Lett., vol. 9, no. 7, pp. 943-947, 2020.

[50] B. F. Beidas, R. I. Seshadri, M. Eroz, and L. Lee, "Faster-than-Nyquist signaling and optimized signal constellation for high spectral efficiency communications in nonlinear satellite systems," in Proc. IEEE MILCOM Conf., 2014, pp. 818-823.

[51] M. El-Hajjar and L. Hanzo, "EXIT charts for system design and analysis," IEEE Commun. Surveys Tuts, vol. 16, no. 1, pp. 127-153, 2014.

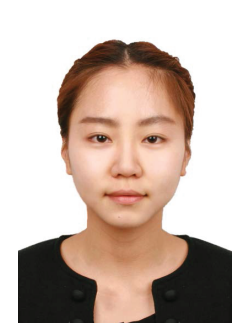

Qiaolin Shi (Student Member, IEEE) received the $\mathrm{Ph} . \mathrm{D}$. degree from the Beijing Institute of Technology, China, and the Ph.D. degree from the University of Technology Sydney, Australia, in 2020. She was a Visiting Ph.D. Student with the University of Southampton, from 2018 to 2019. Her research interests include statistical learning on graphical models and its application to wireless communications. Mrs. Shi has served as a Reviewer for several international journals and conferences.

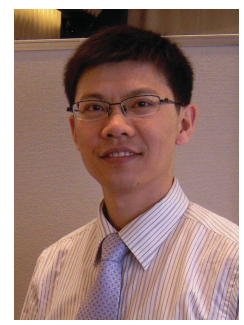

Nan Wu (Member, IEEE) received his B.S., M.S and Ph.D. degrees from Beijing Institute of Technology (BIT), Beijing, China in 2003, 2005 and 2011, respectively. From 2008 to 2009, he was a Visiting Ph.D. Student with the Department of Electrical Engineering, Pennsylvania State University, USA. He is currently a Professor with the School of Information and Electronics, BIT. His research interests include signal processing in wireless communication networks. He was a recipient of the National Excellent Doctoral Dissertation Award by MOE of China in 2013. He serves as an Editorial Board Member of the IEEE Access, the International Journal of Electronics and Communications, the KSII Transactions on Internet and Information Systems, and the IEICE Transactions on Communications. 


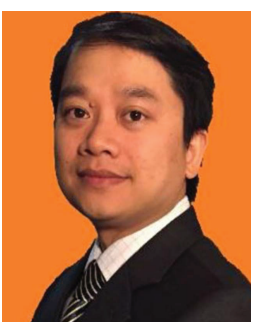

Diep N. Nguyen (Senior Member, IEEE) received the M.E. degree in electrical and computer engineering from the University of California, San Diego (UCSD) and the Ph.D. degree in electrical and computer engineering from The University of Arizona, (UA). He is currently a Faculty Member with the Faculty of Engineering and Information Technology, University of Technology, Sydney (UTS). Before joining the UTS, he was a DECRA Research Fellow with Macquarie University, and a member of Technical Staff with Broadcom, CA, USA, and ARCON Corporation, Boston, consulting the Federal Administration of Aviation on turning detection of UAVs and aircraft, U.S. Air Force Research Laboratory on anti-jamming. His current research interests include computer networking, wireless communications, and machine learning applications, with an emphasis on systems performance and security/privacy. He was the recipient of the several awards from LG Electronics, UCSD, UA, U.S. National Science Foundation, and Australian Research Council. He was the Editor, Associate Editor of the IEEE TRANSACTIONS ON MOBILE COMPUTING, IEEE ACCESS, IEEE SENSORS JOURNAL, and IEEE OPEN JOURNAL OF THE COMMUNICATIONS SOCIETY (OJ-COMS).

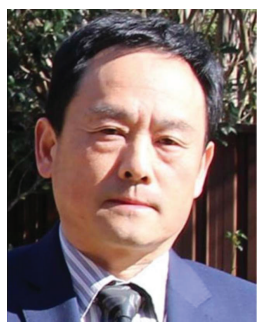

Xiaojing Huang (Senior Member, IEEE) received the B.Eng., M.Eng., and Ph.D. degrees in electronic engineering from Shanghai Jiao Tong University, Shanghai, China, in 1983, 1986, and 1989, respectively. He was a Principal Research Engineer with the Motorola Australian Research Center, Botany, NSW, Australia, from 1998 to 2003, and an Associate Professor with the University of Wollongong, Wollongong, NSW, Australia, from 2004 to 2008. He had been a Principal Research Scientist with the Commonwealth Scientific and Industrial Research Organisation (CSIRO), Sydney, NSW, Australia, and the Project Leader of the CSIRO Microwave and mm-Wave Backhaul projects since 2009. He is currently a Professor of Information and Communications Technology with the School of Electrical and Data Engineering and the Program Leader for Mobile Sensing and Communications with the Global Big Data Technologies Center, University of Technology Sydney (UTS), Sydney, NSW, Australia. His research interests include high-speed wireless communications, digital and analog signal processing, and synthetic aperture radar imaging. With over 32 years of combined industrial, academic, and scientific research experience, he has authored over 330 book chapters, refereed journal and conference papers, major commercial research reports, and filed 31 patents. Prof. Huang was a recipient of the CSIRO Chairmans Medal and the Australian Engineering Innovation Award in 2012 for exceptional research achievements in multigigabit wireless communications.

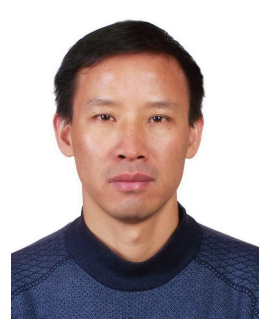

Hua Wang (Member, IEEE) received his Ph.D. degree from Beijing Institute of Technology (BIT), Beijing, China, in 1999. He is now a Professor with School of Information and Electronics, BIT. From Feb. 2009 to Jan. 2010, he was a visiting professor with Department of Electrical Engineering, Arizona State University, USA. His research interests are in the fields of communication theory and signal processing, wireless networking, modem design and implementation for satellite communication.

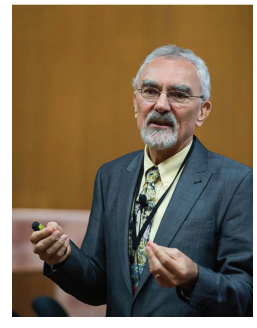

Lajos Hanzo (Fellow, IEEE) received his Master degree and Doctorate in 1976 and 1983, respectively from the Technical University (TU) of Budapest. He was also awarded the Doctor of Sciences (DSc) degree by the University of Southampton (2004) and Honorary Doctorates by the TU of Budapest (2009) and by the University of Edinburgh (2015). He is a Foreign Member of the Hungarian Academy of Sciences and a former Editor-in-Chief of the IEEE Press. He has served several terms as Governor of both IEEE ComSoc and of VTS. He has published 1970 contributions at IEEE Xplore, 19 Wiley-IEEE Press books and has helped the fast-track career of $123 \mathrm{PhD}$ students. Over 40 of them are Professors at various stages of their careers in academia and many of them are leading scientists in the wireless industry. He is also a Fellow of the Royal Academy of Engineering (FREng), of the IET and of EURASIP. 